Cahiers $d u$ MONDE RUSSE

\section{Cahiers du monde russe}

Russie - Empire russe - Union soviétique et États indépendants

\section{$56 / 1 \mid 2015$}

Fictions d'avenir : sciences et temps des socialismes est-européens

\title{
Filmer la nature déchaînée
}

La circulation des images de coulées de boue entre film scientifique, documentaire et film catastrophe au Kazakhstan, 1970-1980

Filming unbridled nature: circulation of mudflow footage between scientific, documentary and disaster films in Kazakhstan, 1970-1980

\section{Marc Elie}

\section{OpenEdition \\ Journals}

Édition électronique

URL : http://journals.openedition.org/monderusse/8164

DOI : 10.4000/monderusse.8164

ISSN : $1777-5388$

Éditeur

Éditions de l'EHESS

Édition imprimée

Date de publication : 1 janvier 2015

Pagination : 25-52

ISBN : 978-2-7132-2475-1

ISSN : $1252-6576$

\section{Référence électronique}

Marc Elie, «Filmer la nature déchaînée ", Cahiers du monde russe [En ligne], 56/1 | 2015, mis en ligne le 13 juillet 2019, consulté le 10 octobre 2020. URL : http://journals.openedition.org/monderusse/8164 ; DOI : https://doi.org/10.4000/monderusse.8164

Ce document a été généré automatiquement le 10 octobre 2020

(c) École des hautes études en sciences sociales 


\section{Filmer la nature déchaînée}

La circulation des images de coulées de boue entre film scientifique, documentaire et film catastrophe au Kazakhstan, 1970-1980

Filming unbridled nature: circulation of mudflow footage between scientific, documentary and disaster films in Kazakhstan, 1970-1980

\section{Marc Elie}

Mes remerciements pour leurs conseils vont à Valérie Pozner, Irina Tcherneva et Fabien Locher.

1 Alma-Ata (aujourd'hui Almaty), capitale du Kazakhstan soviétique, est exposée à des aléas géologiques majeurs. Située dans une zone de forte sismicité, elle fut déjà une fois dans sa courte existence rasée par un tremblement de terre (1887). De plus, la proximité des cimes du Tian Shan place le cœur de la ville à la portée d'avalanches de neige et de glace, de coulées de boue et de glissements de terrain. Ainsi, une vigoureuse coulée détruisit en 1921 les quartiers centraux où quelque 500 personnes trouvèrent la mort ${ }^{1}$. La conscience de la situation très exposée et vulnérable de la ville a mis dès les années 1920 la recherche de protections contre les laves torrentielles ${ }^{2}$ au centre des préoccupations des acteurs politiques, des ingénieurs et des scientifiques.

Dans ce contexte, les résultats des recherches scientifiques sur les coulées étaient des enjeux sociotechniques et politiques. Phénomènes impétueux, dangereux et difficilement observables, les coulées étaient l'objet de disputes scientifiques et de controverses techniques. La direction politique sous le premier secrétaire du parti Dinmuhamed Kunaev avait favorisé dans les années 1960 la construction d'un barrage géant contre les coulées sur le torrent de la Petite Almatinka où s'était formée la coulée de $1921^{3}$. Depuis, la ville était réputée complètement protégée. Or le barrage comme protection contre l'aléa correspondait à une conception hydraulique de la physique des coulées encore dominante dans les années 1940 parmi les spécialistes, mais déjà sérieusement remise en cause à la décennie suivante. En conséquence, des controverses continuaient d'agiter les milieux scientifiques à Alma-Ata dans les années 1970 entre défenseurs et critiques du barrage de Medeo : alors qu'il protégea Alma-Ata contre une coulée en 1973, il paraissait à certains hydrologues inadapté à la nature même de ce phénomène, à ses qualités physiques telles que la recherche récente les exposait. Étant 
donné que Kunaev avait mis son autorité et son crédit dans ce barrage, il tentait au contraire d'imposer un discours approbateur univoque ${ }^{4}$.

3 Au même moment, à l'été 1972, les hydrologues kazakhstanais étaient parvenus à une avancée majeure en produisant artificiellement une coulée de boue dans un chenal naturel, le torrent de Čemolgan. Cette expérimentation fournit de nombreuses photographies et relevés aptes à transformer le regard sur les coulées. Surtout, les hydrologues avaient filmé la coulée expérimentale de 1972 et les suivantes. Ce matériau, destiné d'abord à l'étude de la vitesse et de la composition granulométrique de la coulée, permit de propager son impact visuel bien au-delà du cercle des spécialistes. Avec les retards à la construction du barrage (1961-1972) et son sauvetage in extremis en 1973, la demande en images animées des coulées grandissait chez les réalisateurs pour répondre à des commandes étatiques de justifier le modèle de protection retenu. Les hydrologues fournirent aux studios kazakhstanais les rushes dont les réalisateurs avaient besoin pour rendre visibles les coulées et impressionner le public. Les studios du Kazakhstan ont ainsi produit et diffusé dans les années 1970 pas moins de cinq films sur les coulées de boue à Alma-Ata - un film scientifique, trois documentaires et une fiction long-métrage, dont quatre utilisaient les images et le son enregistrés lors des expérimentations ${ }^{5}$. Les documentaires et la fiction étaient destinés à une large diffusion dans les salles ${ }^{6}$.

4 Le présent article analyse la circulation d'images de coulées dans le Kazakhstan du " socialisme avancé ». Il explique comment et pourquoi les hydrologues tournèrent ces images et les réalisateurs réutilisèrent leurs prises de vue. Les scientifiques produisirent ces images cinématographiques dans le cadre d'une controverse scientifique sur les propriétés physiques des coulées, dont les conséquences pour le débat sur l'utilité du barrage de Medeo étaient fondamentales. À l'inverse, les réalisateurs mobilisèrent et exploitèrent les images cinématographiques dans l'objectif direct de justifier le barrage. Paradoxalement, alors que les expérimentations de Čemolgan tendaient à décrédibiliser le barrage comme réponse de long terme à l'aléa des laves torrentielles, les réalisateurs de films documentaires et de fiction mobilisèrent le matériau cinématographique des chercheurs pour convaincre de l'exact opposé : que Medeo était l'unique rempart contre les forces déchaînées de la nature.

5 Cette étude des expérimentations de Čemolgan et de leur prospérité médiatique au Kazakhstan soviétique croise deux champs d'investigation. Elle est d'une part une contribution à l'histoire des sciences en URSS. Délaissant les relations entre pouvoir politique et savants célèbres qui dominent jusqu'à présent l'historiographie, cet article se penche sur un modeste champ de recherches appliquées dans l'URSS après Stalin : les études interdisciplinaires sur les risques de pente (risque glaciaire, avalanches, hydrologie des torrents et de leurs manifestations dangereuses). Pour imposer leurs conceptions scientifiques controversées à la communauté des chercheurs en risques glaciaires, les hydrologues kazakhstanais ont mis en place une expérimentation extrêmement ambitieuse, analysée ici comme la tentative de créer une " place forte " expérimentale difficilement attaquable par leurs adversaires ${ }^{7}$.

6 D'autre part, le présent article s'arrête sur des développements peu connus dans le cinéma du socialisme finissant. Cette histoire est celle d'emprunts et de prêts d'images destinées à produire un choc esthétique chez les spectateurs à une période où le cinéma soviétique cherche des voies pour toucher et fidéliser un public qui s'érode. Dans un contexte de concurrence télévisuelle et de tassement de la fréquentation des salles, la 
direction politique et artistique du cinéma soviétique encourage le cinéma de genre et son exploitation commerciale : le "film du quotidien" (bytovoj fil'm) et la " thématique contemporaine " (sovremennaja tematika), qui se déclinent en un grand nombre de genres et d'écritures filmiques nouvelles ou renouvelées (comédie romantique, "film de production", satire, mélodrame...) sont particulièrement stimulés ${ }^{8}$. Le film catastrophe, qui apparaît à la fin de la décennie comme une tentative de donner une nouvelle vie au "film de production" via des scènes grandioses et terrifiantes, s'inscrit dans cette quête du succès commercial. Le film catastrophe Un bouclier pour la ville, qui est une commande de la direction républicaine pour justifier sa politique en matière d'aléas naturels et qui utilise l'expérimentation des hydrologues, profite de cet engouement pour les effets visuels spectaculaires.

\section{Un problème de vue ? Inconnues et débats dans les recherches sur les coulées}

7 Dans les années 1930, planificateurs et ingénieurs conçurent des ouvrages d'art pour contenir les coulées dans les régions montagneuses en voie d'industrialisation et d'urbanisation. Ainsi, Alma-Ata disposait déjà d'un plan de protection à la fin des années 1930. Mais avec la guerre, le gouvernement soviétique interrompit ces travaux, qui ne reprirent qu'à la fin des années $1940^{\circ}$. L'Académie des sciences structura les recherches en créant en 1947 une " Commission [pour l'étude] des coulées » (selevaja komissija $)^{10}$. Pluridisciplinaire, la commission comprenait essentiellement trois groupes : des géographes et géomorphologues intéressés à la répartition et à la cartographie du phénomène sur le territoire soviétique ; des ingénieurs des travaux publics concevant des ouvrages d'art protecteurs; des hydrologues et géologues intéressés à la compréhension de la physique des coulées et à leur modélisation mathématique. Les préoccupations des ingénieurs étaient centrales pour la Commission : il s'agissait partout comme à Alma-Ata d'imaginer rapidement des ouvrages pour protéger sites industriels et zones urbaines. Par ailleurs, la prévention des coulées fournissait la meilleure justification des recherches et de leur financement.

Comme le rappelait Mihail Andreevič Velikanov, le premier président de la Commission, pour déterminer quel type d'ouvrage construire, il fallait comprendre la coulée dans ses caractéristiques physiques : en particulier, sa densité était un facteur clef pour savoir s'il fallait lutter contre une manifestation essentiellement hydraulique (une crue pluviale) ou essentiellement géologique (un éboulis de pierres) ${ }^{11}$. Or les coulées étaient alors des phénomènes largement inexpliqués et inexplorés. Les causes et facteurs déclencheurs contribuant à la formation de coulées, leur vitesse, leur composition, leur mode d'écoulement étaient sujets d'hypothèses variées et souvent antagonistes. Les discussions étaient d'autant plus animées à la Commission des coulées que les données quantitatives et les observations qualitatives étaient rares et parcellaires. Le terme " coulée » $\left(s e l^{\prime}\right)$ réunissait alors des phénomènes très différents : de la crue torrentielle au glissement de terrain, en passant par l'avalanche de glace. Les disputes sur la terminologie et la nomenclature dominaient les débats. Les chercheurs, qui n'avaient jamais vu de coulée ou bien en avaient aperçu une par hasard, avaient du mal à s'entendre sur la définition même du phénomène.

9 Pour la plupart des membres de la commission, les coulées n'étaient qu'un type particulier de crues torrentielles (selevoj pavodok), phénomène bien connu et facilement 
observable. Selon cette conception, les coulées étaient composées avant tout d'eau : la différence avec une crue était la présence d'une grande quantité de sédiments et de cailloux dans le mélange, ce qui lui donnait une force destructrice unique ${ }^{12}$. Selon cette conception, des averses diluviennes étaient le facteur conduisant à la formation des coulées dans les hautes vallées érodées à forte inclinaison, typique des chaînes d'Asie centrale et du Caucase. Dans ce paradigme, l'hydrologie et la météorologie étaient les deux disciplines aptes à expliquer la composition et la dynamique du flux et à prévoir les conditions atmosphériques favorables à sa formation. Cette définition des coulées comme " flux d'eau plus matériel érodé » expliquait bien l'événement majeur de 1921. Les colons russes avaient coupé les deux tiers de la forêt qu'ils avaient trouvée en fondant Alma-Ata (Vernyi), provoquant l'accélération des processus érosifs : d'énormes quantités de débris alpins encombraient les vallées ${ }^{13}$. De fortes pluies avaient marqué le début de juillet 1921 : les cours d'eau étaient chargés au point qu'une crue était inévitable. Débris et eau en abondance : le « cocktail» destructeur était servi.

Les travaux de la Commission mirent à mal ce paradigme hydraulique (pavodkovaja koncepcija). Dès sa première réunion en 1949, il apparut que certaines des plus dangereuses coulées contenaient très peu d'eau. Précisément du fait de cette haute densité, leur flux était capable de charrier des blocs de plusieurs tonnes sur de longues distances ${ }^{14}$. De plus, les coulées déformaient le lit des torrents : elles pouvaient les creuser sur plusieurs dizaines de mètres de profondeur. Le président de la commission, Velikanov, remarquait que ces observations défiaient la thèse selon laquelle la coulée était essentiellement une $\mathrm{crue}^{15}$. Au cours des réunions suivantes, la Commission précisa la palette des phénomènes couverts par le terme " coulée » : en fonction de facteurs combinés qu'étaient la vitesse, l'énergie, le débit et la densité, elle classait désormais les coulées en "flux turbulents (ou ruisselants)", (turbulentnye, tekučie potoki), composés essentiellement d'eau chargée de sédiments et se déplaçant selon les lois de l'hydrodynamique et en « flux structurés (ou liés)» (strukturnye, svjaznye potoki), dans lesquels la concentration de sédiments était telle que ce n'était plus l'eau le transporteur, mais les matériaux solides ${ }^{16}$.

11 Une seconde série de données mettait en doute l'explication météorologico-hydrologique. Deux mois avant une quatrième réunion de la Commission à Alma-Ata, lors d'une très belle journée de l'été 1956, une coulée frappa la banlieue nord de la capitale ${ }^{17}$. Le facteur déclencheur ne faisait aucun doute : de la neige fondue provenant du glacier Tujuk-Su qui donnait naissance à la Petite Almatinka. Certes, il était déjà connu que lacs de fonte et moraines glaciaires pouvaient déclencher des coulées en rejetant brutalement l'eau qu'ils retenaient. Mais les coulées d'origine glaciaire étaient réputées moins dangereuses, car supposément moins volumineuses que les coulées pluviales ${ }^{18}$. Or, en 1956, les dégâts avaient été très importants. Il apparaissait qu'un lac de moraine contenant tout au plus quelques dizaines de milliers de mètres cubes d'eau pouvait déclencher dans un chenal sec une coulée dont le volume se comptait en millions de mètres cubes. Jusqu'alors, la coulée étalon était celle de 1921, qu'un volume considérable accumulé rapidement par des averses diluviennes avait déclenchée. L'événement de 1956 troublait et inquiétait les spécialistes : premièrement, s'il était déjà difficile de prévoir des coulées d'origine pluviale, que dire alors des coulées qui naissaient à 3 500-4 000 mètres d'altitude dans les glaciers ? Un risque sporadique difficilement prévisible menaçait toutes les villes et infrastructures de la région d'Alma-Ata. Deuxièmement, la reforestation et la 
protection du couvert forestier, ces mesures unanimement reconnues comme bénéfiques pour lutter contre l'érosion, étaient insuffisantes pour prévenir les coulées glaciaires qui naissaient au-dessus de la limite supérieure de la forêt. Troisièmement, la formation de coulées de boue était profondément contre-intuitive : elles se déclaraient par beau temps dans les lits secs de torrent à faible débit. Et alors qu'elles avaient l'aspect de sols en mouvement, elles déployaient, contrairement aux glissements de terrain, des vitesses considérables ${ }^{19}$.

12 Les tâtonnements des spécialistes s'expliquaient avant tout par la dangerosité et la relative rareté dans un même bassin des coulées de boue : assister et réchapper à une coulée naturelle relevait soit de la chance, soit d'une observation minutieuse et systématique sur plusieurs années d'un chenal particulièrement exposé. Les stations météorologiques et hydrologiques traditionnelles étaient inadaptées à l'observation des coulées, qui arrachaient leurs systèmes de mesure disposés en travers des cours d'eau. Il fallait créer et tester de nouveaux instruments pour remplacer les instruments d'hydrométrie (densitomètre, jauge de profondeur, débitmètre...). Il y avait donc un problème tout à la fois de vision et de visualisation : il était non seulement difficile de voir la coulée de ses propres yeux, mais aussi de la mesurer afin d'en créer des représentations graphiques lisibles (hydrogrammes, profils géodésiques) ${ }^{20}$.

\section{Visualiser l'inconnu}

13 Le premier documentaire sur les coulées au Kazakhstan date de $1970^{21}$. Sa fonction politique était nettement exprimée : il s'agissait de justifier rétrospectivement la construction du barrage anti-coulée de Medeo, dont l'opportunité avait divisé l'intelligentsia kazakhstanaise entre 1958 et 1965. Au moment où Kulakov tournait son film, le barrage n'était pas encore terminé : il fallut encore deux ans aux maçons pour achever les travaux dans l'urgence ${ }^{22}$. Mais l'acte essentiel, auquel les scientifiques spécialistes des coulées et les protecteurs des montagnes s'étaient vigoureusement opposés, était déjà commis : en 1966 et 1967, deux gigantesques explosions avaient effondré les versants de la vallée de la Petite Almatinka à l'encaissement dit de Medeo afin de former un bouchon de roche censé arrêter les coulées de boue dans leur course sur la capitale.

14 C'est donc moins un souci de prévention qui conduisit le réalisateur à consacrer une dizaine de minutes sur un documentaire qui en compte cinquante à la description des malheurs apportés par les coulées, que la volonté de présenter rétrospectivement les explosions comme nécessaires. Le film était d'ailleurs une commande de l'agence kazakhstanaise des explosions de génie civil, Kazvzryvprom. Le titre, "L'explosion créatrice » résume l'ambition du film de présenter les explosifs, détournés de leur fonction destructrice et guerrière, comme des forces positives dans les travaux de terrassement des chantiers les plus prestigieux pour accélérer la transformation de la nature et la construction du communisme. L'agence fournit trois consultants au réalisateur, dont deux ingénieurs préparateurs des explosions de 1966-1967. L'explication scientifique qu'ils donnaient de l'origine et de la nature des coulées s'inscrivait dans le cadre du paradigme hydraulique.

15 Quelles images montrer pour frapper les esprits ? Kulakov n'a pas d'enregistrement de coulée. Il procède donc à un montage d'archives qu'il équipe d'un fond sonore destiné à plonger le spectateur dans une catastrophe familière. Conformément à la 
représentation que ses conseillers scientifiques se font des coulées, il prend des enregistrements de crues catastrophiques : des crues de montagne, des crues de plaine. C'est un bric-à-brac d'actualités étrangères et d'images tournées en URSS : on y trouve même les images fameuses de la rupture du Baldwin Hills Dam à Los Angeles en 1963 filmées en direct par des reporters de KTLA ${ }^{23}$. Le montage de Kulakov place d'abord les spectateurs face à d'irrésistibles forces météorologiques : de violents orages strient le ciel d'éclairs et déversent de puissantes averses. L'eau ruisselle depuis les sommets. Une voix hors-champ indique d'un ton posé, mais sur une musique inquiétante, que « les eaux féroces, emportant avec elles la masse toujours croissante des fragments rocheux se ruent vers l'aval avec une force folle et indomptable" et que " les chaudes pluies estivales sont toujours un facteur intensifiant le danger ». La crue enfle terriblement et finit par tout inonder dans les villes d'aval. Le spectateur est alors plongé dans la désolation : des panoramas et lents travellings découvrent des paysages de plaines englouties. Des sinistrés désemparés s'agglutinent dans des abris de fortune ou prennent la route en hâte pour fuir le cataclysme, abandonnant leurs maisons, chargeant femmes et enfants et de maigres biens sur des chariots. Ces images de déluge rappellent irrésistiblement les ravages de la guerre et l'exode des populations civiles (Alma-Ata avait accueilli de nombreux évacués pendant la Grande Guerre patriotique) [Ill. 1 et 2].

Le montage de Kulakov ne reflète ni ce qu'est une coulée de boue telle qu'on la redoutait à Alma-Ata, ni les dégâts qu'elle avait effectivement déjà suscités au Kazakhstan. Les coulées de boue n'inondent pas les plaines à l'image des grandes crues printanières. Les destructions causées par les coulées ne sont pas de celles qu'on voit dans les débâcles : les problèmes sont les pluies de rochers et les coups portés par les vagues de boue aux infrastructures. L'accompagnement sonore plonge le spectateur dans un monde de perceptions très éloigné de celui d'une coulée de boue : Kulakov a monté le bruit de l'eau ruisselante sur les images d'archives. Or, la coulée ne ruisselle pas, elle fait trembler la terre et produit un terrible fracas que les témoins auriculaires des coulées au Kazakhstan avaient déjà décrit. Ni le bruit, ni l'aspect de la coulée ne rappellent l'écoulement même torrentiel de l'eau. En étendant la géographie des dégâts des coulées à toute la planète, Kulakov atteint toutefois son objectif de "vendre » les explosions comme une prouesse technique absolument nécessaire pour épargner à la capitale les épreuves de la guerre.

Cette représentation de la coulée correspondait au paradigme hydraulique, largement rejeté par les chercheurs mais encore dominant chez les ingénieurs au moment du tournage. Si la coulée était bien essentiellement un cours d'eau chargé de cailloux, alors une levée plaine, dotée à sa base d'un tunnel devait faire l'affaire : le rempart retiendrait les rochers, et l'eau filtrerait par le conduit. C'était le schéma prévu pour Medeo $^{24}$. Le film de Kulakov liait fermement trois assertions : la conception hydraulique des coulées, la nécessité d'un barrage pour y faire face et celle d'explosions massives pour le bâtir.

\section{Voir l'insaisissable : L'expérimentation de Čemolgan}

Étant donné la difficulté à observer des coulées, les produire artificiellement en laboratoire ou dans un chenal naturel paraissait une voie nécessaire pour la recherche. Les chercheurs abordaient certes les coulées à partir de leurs traces : creusement des 
chenaux, dépôts sédimentaires. Mais cette méthode reposait sur un repère de densité à partir duquel on calculait vitesse, débit, force d'impact, etc. Or, c'est précisément la densité des coulées qui était l'objet des débats ${ }^{25}$. En fonction de leurs convictions plus ou moins « hydrauliques », les chercheurs extrapolaient à partir d'une densité basse ou d'une densité haute. Les conséquences pratiques de ce choix sur les paramètres de la coulée étaient énormes : d'une densité assez élevée de $1800 \mathrm{~kg} / \mathrm{m}^{3}$ à une densité très élevée de $2450 \mathrm{~kg} / \mathrm{m}^{3}$, le débit et le volume de la coulée sont quintuplés ${ }^{26}$.

Au début des années 1950, un géographe de l'Institut de météorologie d'Alma-Ata, Semen Parfenovič Kaveckij (1912-1974), expérimenta dans la vallée du Čimbulak au-dessus d'Alma-Ata. Avec l'aide d'un ingénieur, Ivan Pavlovič Smirnov (1899-?), il avait fait construire un petit barrage simple pour accumuler l'eau nécessaire au déclenchement de la coulée. En ouvrant la vanne du barrage, les chercheurs produisaient une brusque arrivée d'eau dans la vallée. L'eau se chargeait de sédiments et de débris, le flux s'épaississait et ravageait les petites digues construites en travers du lit du torrent ${ }^{27}$. Kaveckij et Smirnov avaient filmé les expériences afin de calculer la vitesse des coulées. Ils en firent un film scientifique ${ }^{28}$. Malgré le scepticisme initial des collègues à la Commission des coulées, Kaveckij et Smirnov avaient franchi un pas décisif dans les techniques d'expérimentation et ouvert des perspectives nouvelles pour l'étude ${ }^{29}$.

21 Un collègue de Kaveckij, Jurij Borisovič Vinogradov (1932-2012), arrivé au Kazakhstan de Taškent à la fin des années 1950, reprit ces expériences vingt ans plus tard. Après avoir observé plusieurs années de suite des coulées naturelles sur la rivière Kokčeta, dans la région d'Alma-Ata, l'ambitieux hydrologue s'était convaincu que les coulées de forte densité progressaient par glissement (cisaillement, zdvigovyj process), et non pas par écoulement : la coulée démarre quand la masse de roches et de sédiments couvrant le lit d'un torrent est imprégnée d'eau au-delà d'un seuil critique. Mais l'eau ne transporte pas sédiments et roches : ils se mettent en branle sous l'effet de la contrainte de cisaillement qui leur est appliquée. Il avait adopté, de son propre aveu par esprit de contradiction, le point de vue diamétralement opposé à la conception hydraulique, qui faisait de toutes les coulées des cours d'eau chargées de sédiments ${ }^{30}$. Dès la fin des années 1960 il cherchait à produire une expérimentation qui confirmât ce mode de déplacement des coulées. Pour lui, les résultats expérimentaux de Kaveckii étaient décevants : la masse volumique des coulées obtenues n'était que marginalement supérieure à celle de l'eau $\left(1200 \mathrm{~kg} / \mathrm{m}^{3}\right)$, le chenal était trop court $(500 \mathrm{~m})$, la pente, trop faible (15\%), et les matériaux d'érosion, trop peu nombreux ${ }^{31}$. Kaveckij et Smirnov n'avaient obtenu que de l'eau de crue, qui s'accordait bien à la conception hydraulique des coulées qu'ils partageaient, mais pas à la mise en mouvement de matériaux érosifs que voulait décrire Vinogradov.

Pour Vinogradov, la classification traditionnelle des coulées était insatisfaisante ${ }^{32}$ : elle focalisait l'attention sur des phénomènes intermédiaires de faible densité parmi lesquels il était difficile de systématiser les caractères physiques typiques des coulées étant donné la dispersion des données sur une grande échelle de phénomènes et la difficulté à les distinguer les uns des autres ${ }^{33}$. Vinogradov entendait renverser la perspective en concentrant son attention sur les phénomènes du bout, et même au-delà de l'échelle classique : il pensait trouver le mécanisme central de fonctionnement des coulées en analysant des phénomènes de densité exceptionnelle. Les densités maximales ne seraient plus alors les confins marginaux d'une science qui se concentre 
sur les phénomènes les plus courants - des flux relativement liquides - mais le cœur de la discipline. L'échelle de classification des coulées selon la densité serait par là même étendue vers le haut : au lieu d'un maximum autour de $2000-2100 \mathrm{~kg} / \mathrm{m}^{3}$ de masse volumique on déclarerait typiques des coulées à $2300 \mathrm{~kg} / \mathrm{m}^{3}$, et on placerait les maxima à plus de $2600 \mathrm{~kg} / \mathrm{m}^{3}$. Vinogradov voulait produire une expérience cruciale pour démontrer que les coulées pouvaient atteindre des densités extrêmes et que les contraintes de cisaillement étaient les principaux facteurs de mise en mouvement des débris alpins qui jonchaient le lit des torrents ${ }^{34}$.

$\mathrm{Au}$ sein de l'Institut de météorologie (KazNIGMI) de l'agence de météorologie kazakhstanaise (Kazgidromet) fonctionnait un secteur des coulées animé par quelques enthousiastes autour de Kaveckij dans les années 1940-1950. Sous la direction de Vinogradov à partir de 1964, le secteur des coulées compta bientôt quatre-vingts chercheurs, ingénieurs et techniciens ${ }^{35}$. Vinogradov profita dans sa carrière scientifique de l'appel d'air créé par l'implication des plus hautes figures du pouvoir dans la problématique du barrage. Il se révéla un organisateur déterminé : en deux ans (1971-1972) il mit en place une expérience grandeur nature bien plus ambitieuse que celle qu'avait réussie en son temps Kaveckij. S'appuyant pratiquement sur les seules forces internes du laboratoire, il fit dresser un barrage en béton de $4,5 \mathrm{~m}$ de haut pour créer un réservoir de $73000 \mathrm{~m}^{3}$ en amont du torrent Čemolgan, à $2900 \mathrm{~m}$ d'altitude. Situé à $80 \mathrm{~km}$ d'Alma-Ata, le lit du Čemolgan était régulièrement et profondément travaillé par des coulées et jonché de matériaux érosifs de tailles diverses. La section du torrent choisie faisait $2200 \mathrm{~m}$ de long, avec une inclinaison moyenne de $30 \%$ dans la partie haute. Comme le barrage en bois de Kaveckij et Smirnov, celui de Vinogradov était réutilisable : des vannes permettaient de faire passer la quantité d'eau au débit voulu (de 50 à $\left.70 \mathrm{~m}^{3} / \mathrm{s}\right)^{36}$. Vinogradov put ainsi réaliser cinq expériences entre 1972 et 1976.

24 L'hydrologue avait voulu faire de l'expérience à Čemolgan un spectacle. Cent cinquante invités - scientifiques de toute l'URSS et notables locaux - étaient présents le 27 août 1972 pour voir l'eau du réservoir se transformer en coulée. La presse écrite et la radio couvraient l'événement. L'hydrologue risquait gros, car il n'avait aucun moyen de prévoir comment interagiraient le volume d'eau brutalement relâché dans le chenal et les débris rocheux qui le jonchaient. Ses hypothèses sur le déplacement des coulées pouvaient être contredites, et elles le furent en partie. De plus, l'expérience pouvait tout simplement " rater ", l'eau ne produisant qu'un petit torrent boueux indigne du nom de « dragon noir » que les spécialistes donnaient au phénomène.

L'expérience fut un succès remarqué : le spectacle était grandiose. Vinogradov monta un film documentaire à la fin des années 1970 à partir des rushes tournés lors de cette expérience et des suivantes. Voici le commentaire de l'hydrologue, où il exprime sa fascination pour une manifestation exceptionnelle de la nature et sa faculté à transporter le spectateur :

La masse de rochers, d'eau et de boue avance dans le vacarme et le grondement. La puissance de la coulée, son souffle lourd, sa sombre beauté provoquent de bouleversantes émotions. Ce monstre noir progresse sans fin, semble-t-il, parfois par bonds énormes, étendant d'aveugles tentacules et modifiant sans cesse ses traits. $^{37}$

Cette expérience et les suivantes produisirent des coulées très denses et néanmoins extrêmement turbulentes : la tête de la coulée bondissait furieusement dans un immense vacarme, éjectant des pierres et enrobée d'un fin nuage de poussière. Le débit 
de la coulée était sept fois plus important que celui de l'eau au sortir des vannes du barrage $\left(120 \mathrm{~m}^{3} / \mathrm{s}\right.$ contre $\left.16 \mathrm{~m}^{3} / \mathrm{s}\right)$. Vinogradov avait isolé un type de coulée auparavant mal décrit en URSS : les laves torrentielles (grjazekamennyj potok). Le flux des coulées expérimentales à Čemolgan n'était pas un glissement par cisaillement comme à Kokčeta. Pour Vinogradov désormais, la coulée progressait selon un schéma intermédiaire entre le glissement par cisaillement des débris rocheux et le transport de matériaux (transportno-sdvigovyj process). L'échelle classique apparaissait inadéquate : les coulées typiques, au moins dans les vallées au-dessus d'Alma-Ata, avaient des densités énormes, jusqu'à $2500 \mathrm{~kg} / \mathrm{m}^{3}$.

L'expérimentation de 1972 et les suivantes permirent à Vinogradov de créer une forteresse expérimentale : on pense aux " places fortes » de Bruno Latour ${ }^{38}$. L'équipe de Vinogradov avait conçu et fabriqué des appareils de mesure des caractéristiques physiques de la coulée. Ces innovations instrumentales, qui montrèrent leur efficacité lors des expériences, firent passer l'observation quantitative des coulées à un autre niveau : en particulier, le " fluxomètre " (selemer), conçu et breveté par un chercheur du laboratoire, Boris Sergeevič Stepanov, permettait d'établir de manière précise la densité des coulées. Les chercheurs étaient ainsi en mesure de produire des hydrogrammes qui rendaient les données visualisables, simplifiables et comparables : tout à coup, les coulées devenaient des phénomènes compréhensibles et réductibles aux procédés de visualisation classique de l'hydrologie ${ }^{39}$. Les données quantitatives amassées à Čemolgan faisaient pâlir les observations antérieures. Vinogradov jugeait ces dernières purement "spéculatives", déduites d'observations qualitatives et colportées d'un auteur à l'autre sans vérification instrumentale. Dispersées, imprécises, parfois farfelues et souvent contradictoires, elles devaient céder le pas à la mesure rigoureuse permise par le dispositif qu'il contrôlait sur le Čemolgan ${ }^{40}$.

L'observation à l'œil nu et le visionnage du film tourné par Vinogradov ne pouvaient trancher en eux-mêmes la controverse sur les densités. Le caractère étonnamment dense des coulées n'apparaissait pas à l'œil nu, même chez un spécialiste exercé. En ce sens, l'expérimentation était un spectacle créant l'illusion d'exposer les caractéristiques physiques du phénomène. Un ingénieur travaillant à l'élaboration du barrage de Medeo pour l'agence Kazgidroproekt, A. Degovec, assista à l'expérimentation et en fournit un enthousiaste rapport :

Le spectacle de ce phénomène était grandiose et impressionnant, et même émotionnellement terrifiant, telle une terrible calamité naturelle ébranlant les versants - le fracas et le crissement des rochers envahissaient tout. ${ }^{41}$

Ses yeux virent une densité de $1700-1800 \mathrm{~kg} / \mathrm{m}^{3}$ (40 à $50 \%$ de sédiments dans le mélange), alors que Vinogradov avait calculé rétrospectivement une densité moyenne de $2070 \mathrm{~kg} / \mathrm{m}^{342}$. La coulée se déplaçait par pulsations. Degovec la rangeait donc parmi les flux turbulents non-liés. Pour Degovec, la coulée expérimentale ne remettait en rien ses connaissances théoriques ni son expérience personnelle en question : "L'expérimentation réalisée par KazNIGMI [...] confirme entièrement les principes [...] élaborés par Kazgidroproekt [...] pour le calcul des paramètres des coulées sur les rivières du Kazakhstan. » Degovec affirmait que la coulée expérimentale de Čemolgan était « proche par ses caractéristiques de [la] coulée naturelle [de 1956] » : cela était exact, mais à des densités bien plus élevées ${ }^{43}$. Avec cette analyse, que d'autres ingénieurs travaillant sur le barrage partageaient, comme nous l'avons vu avec L'explosion créatrice, Degovec pouvait se féliciter du barrage de Medeo. 
30 En revanche, si Vinogradov avait raison, alors le barrage n'était que de peu d'utilité : il retiendrait une coulée de taille moyenne, mais ne pourrait pas en supporter une seconde. En effet, l'épais mélange cimenterait la retenue, y compris l'entrée du tunnel. C'est bien ce qui se passa un an plus tard, en juillet 1973, quand une coulée de boue d'origine naturelle avait été arrêtée par le barrage géant construit sur la Petite Almatinka. La capitale avait échappé de peu à la catastrophe. Il fallut à un coût faramineux évacuer d'urgence la boue qui menaçait de déborder du barrage de Medeo, puis excaver des années durant la retenue où les millions de mètres cubes de mélange avaient sédimenté, afin de la rendre à nouveau efficace contre une future coulée ${ }^{44}$.

31 Les chercheurs du laboratoire de Vinogradov avaient trouvé que cette coulée naturelle montrait des caractéristiques physiques très proches de celles qu'ils avaient lues dans l'expérimentation de Čemolgan, même si les deux coulées se distinguaient par l'échelle : la coulée naturelle de 1973 était cent fois plus importante que la coulée contrôlée des hydrologues ${ }^{45}$. Ils purent montrer que les spécialistes avaient jusqu'alors sérieusement sous-estimé la densité de la plupart des coulées à partir de leurs empreintes dans les vallées ${ }^{46}$. Lorsqu'une coulée frappa Alma-Ata en 1950, les chercheurs envoyés sur place étudier ses traces partirent de l'hypothèse d'une masse volumique de $1300 \mathrm{~kg} / \mathrm{m}^{3}$ avec un volume d'eau d'1,1 million $\mathrm{m}^{3}$. De là, ils avaient calculé un volume du "flux solide » de $300000 \mathrm{~m}^{3}$, un total de la "crue " de 1,4 million $\mathrm{m}^{3}$ et un débit de $970 \mathrm{~m}^{3} / \mathrm{s}$. Comme Jafjazova le démontra des décennies plus tard, la masse volumique de cette coulée était en fait proche de $2400 \mathrm{~kg} / \mathrm{m}^{3}$, et il aurait donc fallu déduire un volume quatre fois supérieur de 6,3 à 7,5 millions $\mathrm{m}^{3}$ débité à $4000-4800 \mathrm{~m}^{3} / \mathrm{s}^{47}$.

32 En 1972, les chercheurs du laboratoire de Vinogradov n'avaient pas pu mesurer la densité de la coulée : celle-ci avait fracassé les débitmètres disposés le long du chenal. Ils avaient fait des relevés de la boue de surface avec des seaux, sur les bords de la coulée, en aval de la section principale, où l'inclinaison est moins forte : ils avaient trouvé seulement $1500 \mathrm{~kg} / \mathrm{m}^{348}$. Bref, la coulée expérimentale "n'affichait » pas ses paramètres physiques. Ce n'est qu'avec les inscriptions permises par l'instrumentation que Vinogradov put confirmer les hautes densités dans les expériences ultérieures.

Quelque chose, pourtant, avait changé dès 1972. Pour la première fois, un nombre important de spectateurs avaient assisté à une lave torrentielle. Surtout, comme Kaveckij avant lui, Vinogradov avait filmé la coulée. Pas moins de 16 caméras étaient en action en 1972, qui tournaient en 35 millimètres couleur. L'une d'elle était montée à bord d'un hélicoptère. Le studio national Kazakhfil'm avait prêté les caméras et leurs opérateurs. Les caméras mesuraient la vitesse de la coulée et permettaient de vérifier sa composition granulométrique ${ }^{49}$. Mais en fait, les caméras permirent surtout à Vinogradov de frapper un grand coup dans le petit monde des coulées en mettant en circulation dans les médias des images saisissantes.

\section{Le film factuel à la rescousse du documentaire}

Trois ans après Kulakov, Asylbek Nugmanov reprit la thématique des coulées dans un film documentaire. Il avait jusqu'alors tourné des documentaires sur l'histoire et les paysages du Kazakhstan. Medeo, jours et nuits de courage chante les héros qui, en juillet 1973, participèrent au sauvetage du barrage de Medeo assailli par une coulée de boue $^{50}$. Envoyé comme reporter sur les lieux, Nugmanov réalisa dans les semaines 
suivant la catastrophe un court documentaire (vingt minutes) destiné à justifier le barrage mis en danger par la coulée de juillet 1973 et à rassurer la population sur la capacité de la direction politique kazakhstanaise à maîtriser les risques venus des montagnes. Il promouvait la " lutte contre les forces déchaînées de la nature ». Cette rhétorique guerrière, qui avait fait florès, était déjà passablement usée dans les années $1970^{51}$.

35 Mais Nugmanov n'avait pas d'image de la coulée en action. Il était arrivé sur les lieux après que l'alerte avait été donnée. Il avait filmé le niveau de la boue montant dangereusement dans la retenue derrière le barrage. Mais à quoi avait ressemblé la coulée quand elle dévalait les pentes ? Les images d'archives abondamment utilisées par Kulakov dans L'explosion créatrice ne faisaient plus l'affaire : il était clair que la coulée n'avait pas l'aspect d'une crue torrentielle, on voyait la boue épaisse dans la retenue. La coulée s'était déclarée par une belle journée d'été. D’ailleurs, Nugmanov avait filmé la station touristique Gorel'nik, ravagée par la coulée de juillet 1973 : on y voyait des bâtiments coupés en deux ou aplatis sous les rochers [Ill. 4]. Rien à voir donc avec les scènes d'exode et les plaines inondées présentées par Kulakov trois ans plus tôt.

\section{1 - Conséquence supposée des coulées : l'inondation des plaines d'aval.}

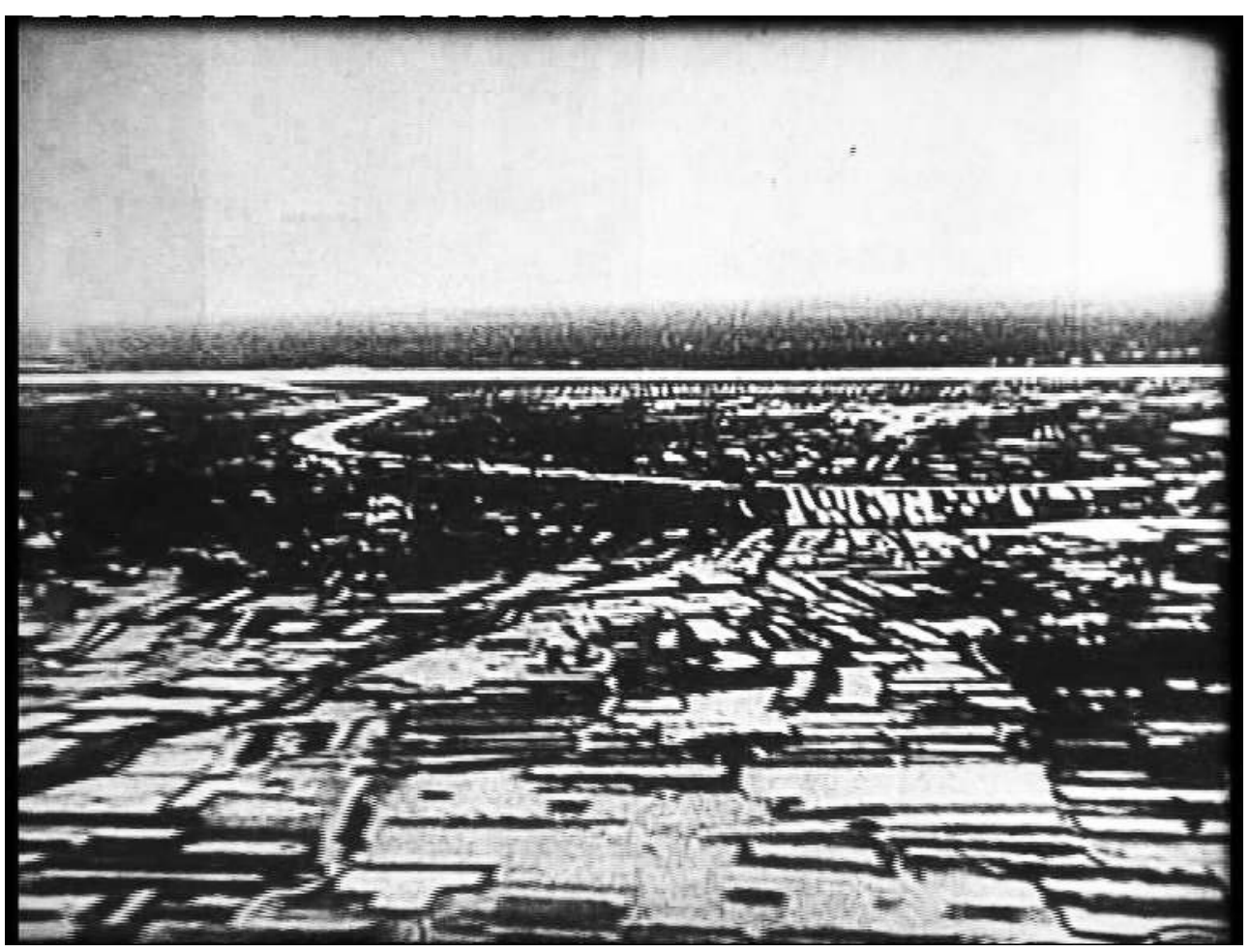

Scène du film Vzryv sozidatel' [L'explosion créatrice]

CGA Kinofotodokumentov i zvukozapisi RK, 1970. 
2 - Conséquences supposées des coulées : l'exode.

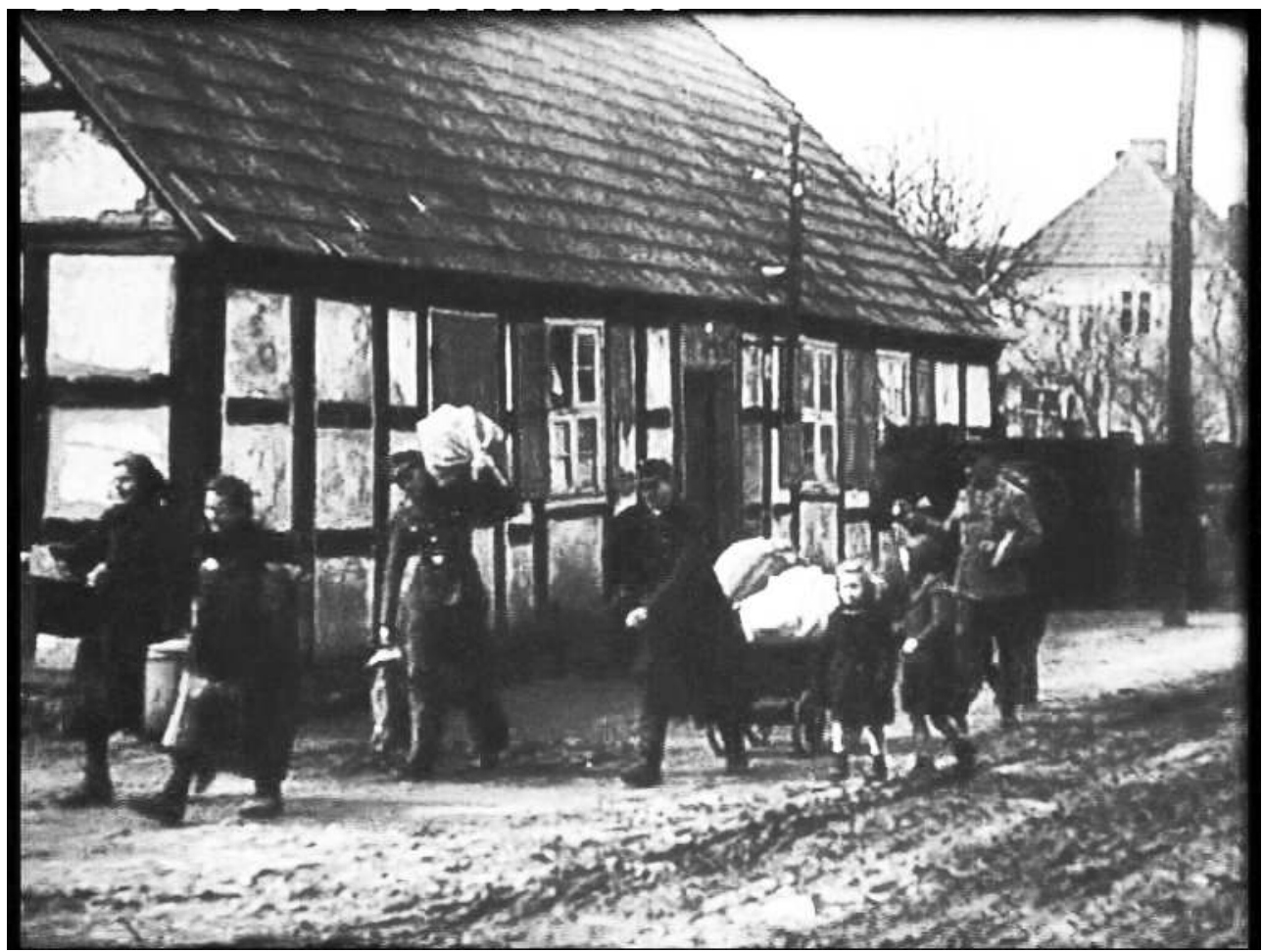

Scène du film Vzryv sozidatel' [L'explosion créatrice] CGA Kinofotodokumentov i zvukozapisi RK, 1970.

3 - Montre au verre cassé, arrêtée sur $18 \mathrm{~h} 12$.

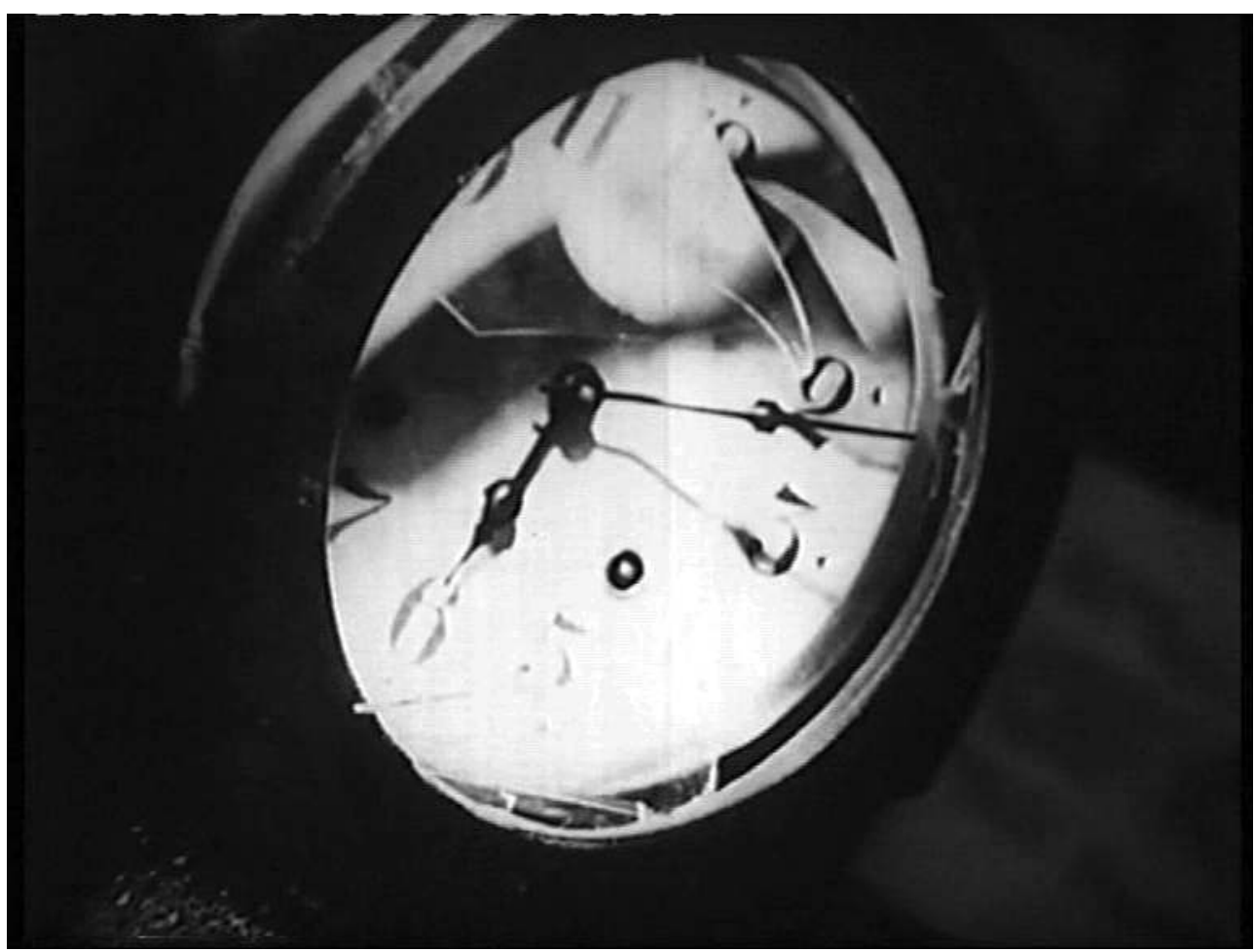

Scène du film Medeo. Dni i noči mužestva [Medeo. Jours et nuits de courage] CGA Kinofotodokumentov i zvukozapisi RK, 1973. 
4 - Bâtiment détruit par la coulée de juillet 1973 à la station alpine de Gorel'nik.

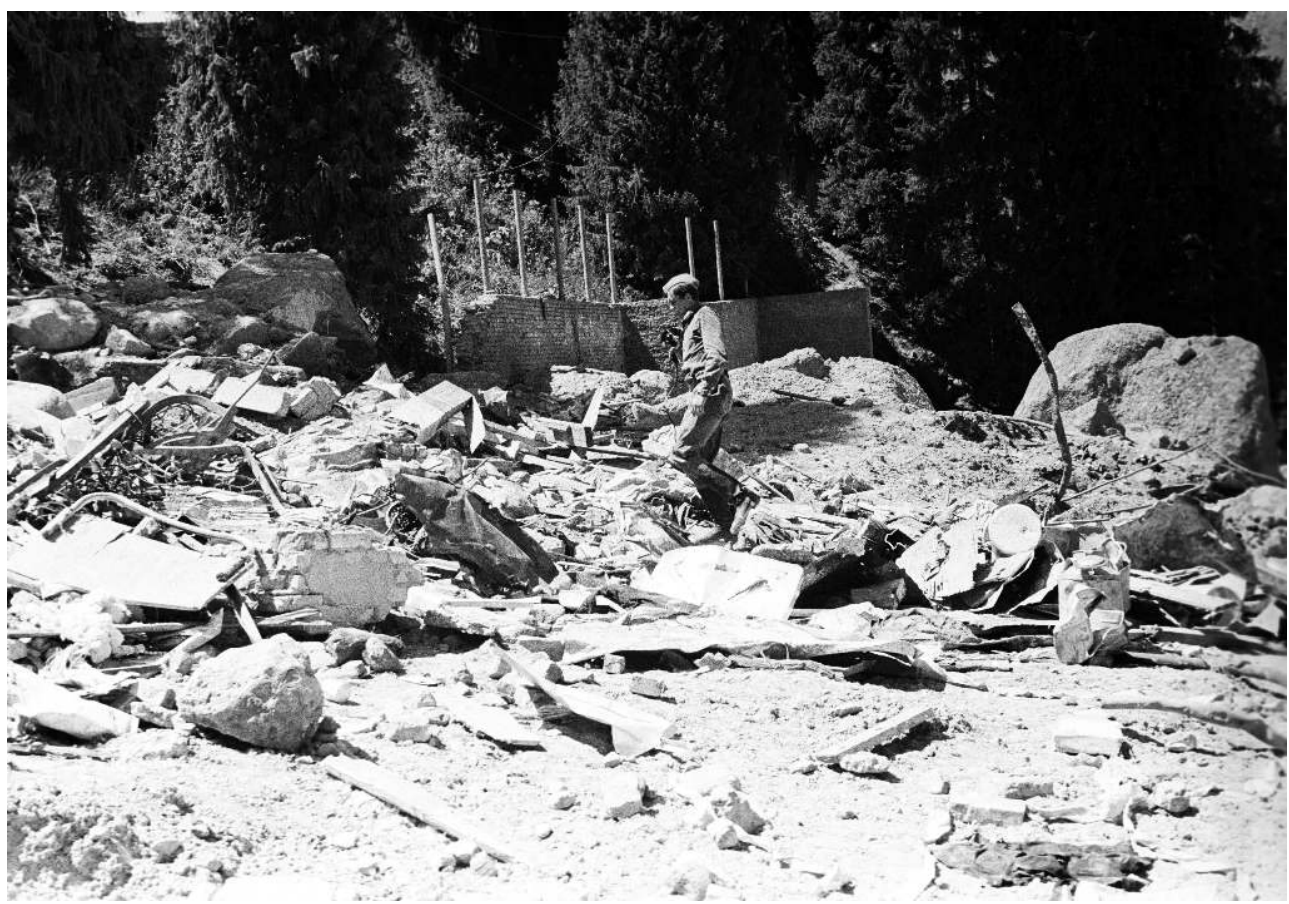

CGA Kinofotodokumentov i zvukozapisi RK n²-71143, 1973.

\section{5 - Expérimentation de Čemolgan}

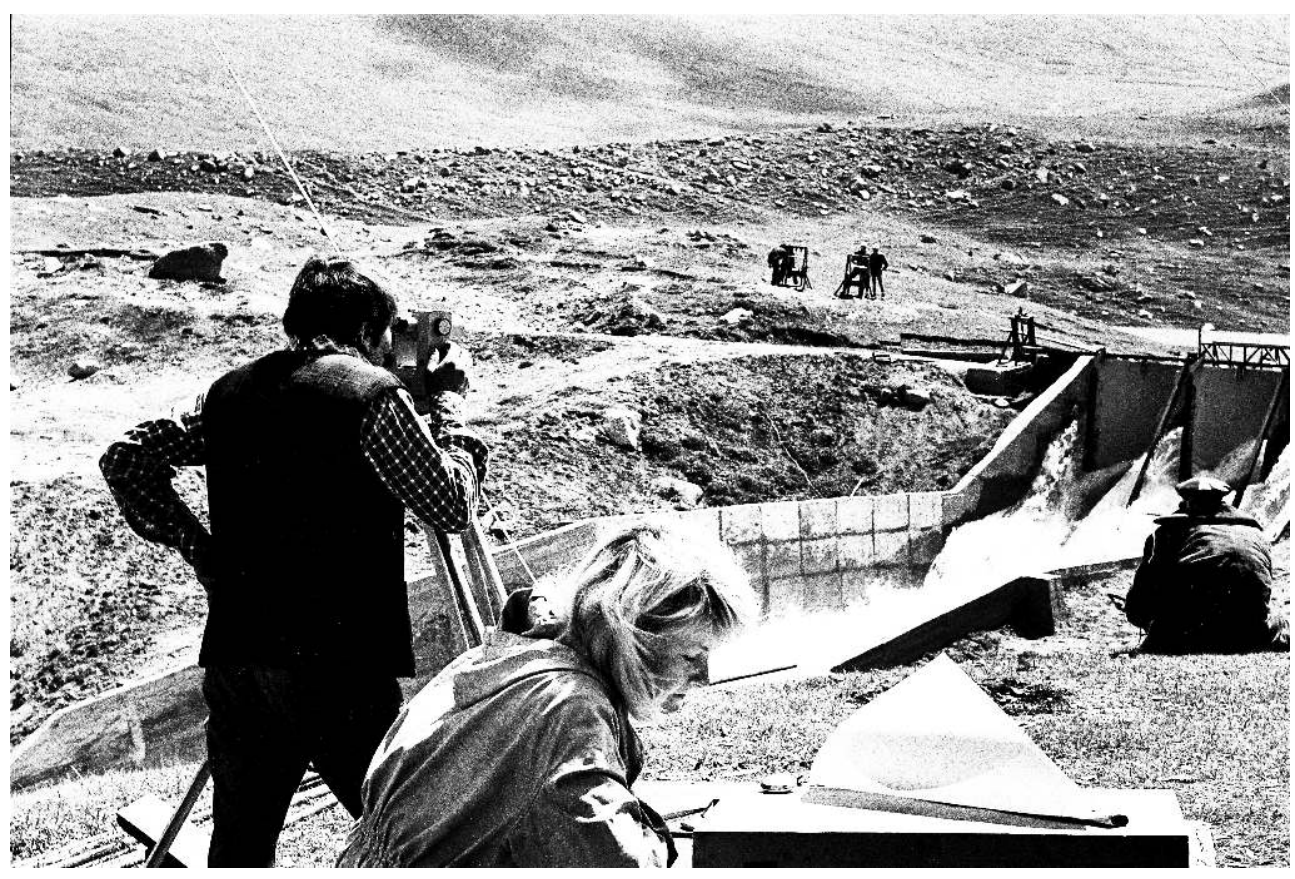

A. Hajdarov et N. Vozovikova observent le déversement du réservoir.

Don d'Alim Hajdarov, archives personnelles, 1975 


\section{6 - Expérimentation de Čemolgan}

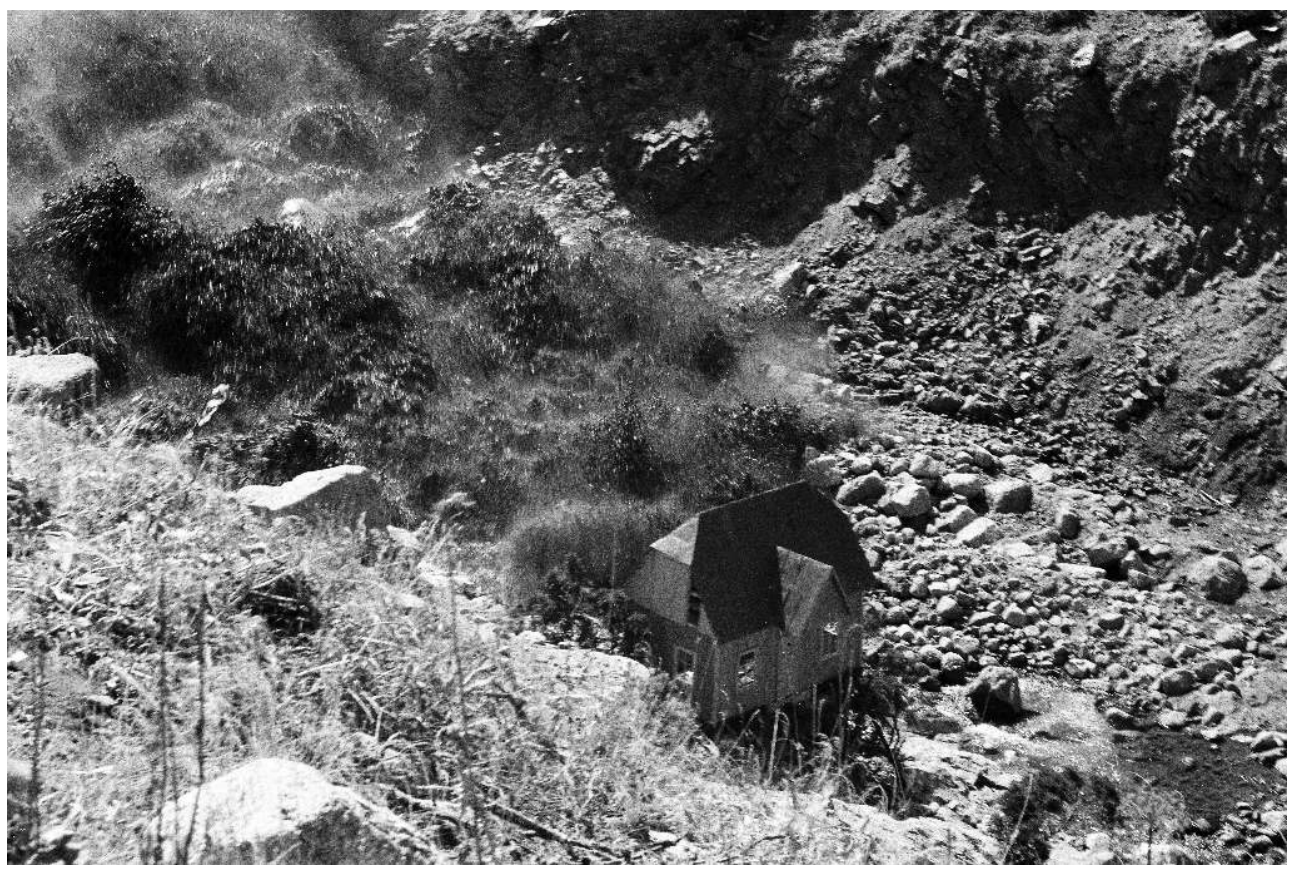

La tête de la coulée atteint les maquettes placées dans le lit pour le tournage de Ščit goroda [Un bouclier pour la ville]

CGA Kinofotodokumentov i zvukozapisi RK, collection personnelle d'Alim Hajdarov, n E-5150, 1978

\section{7 - Expérimentation de Čemolgan}

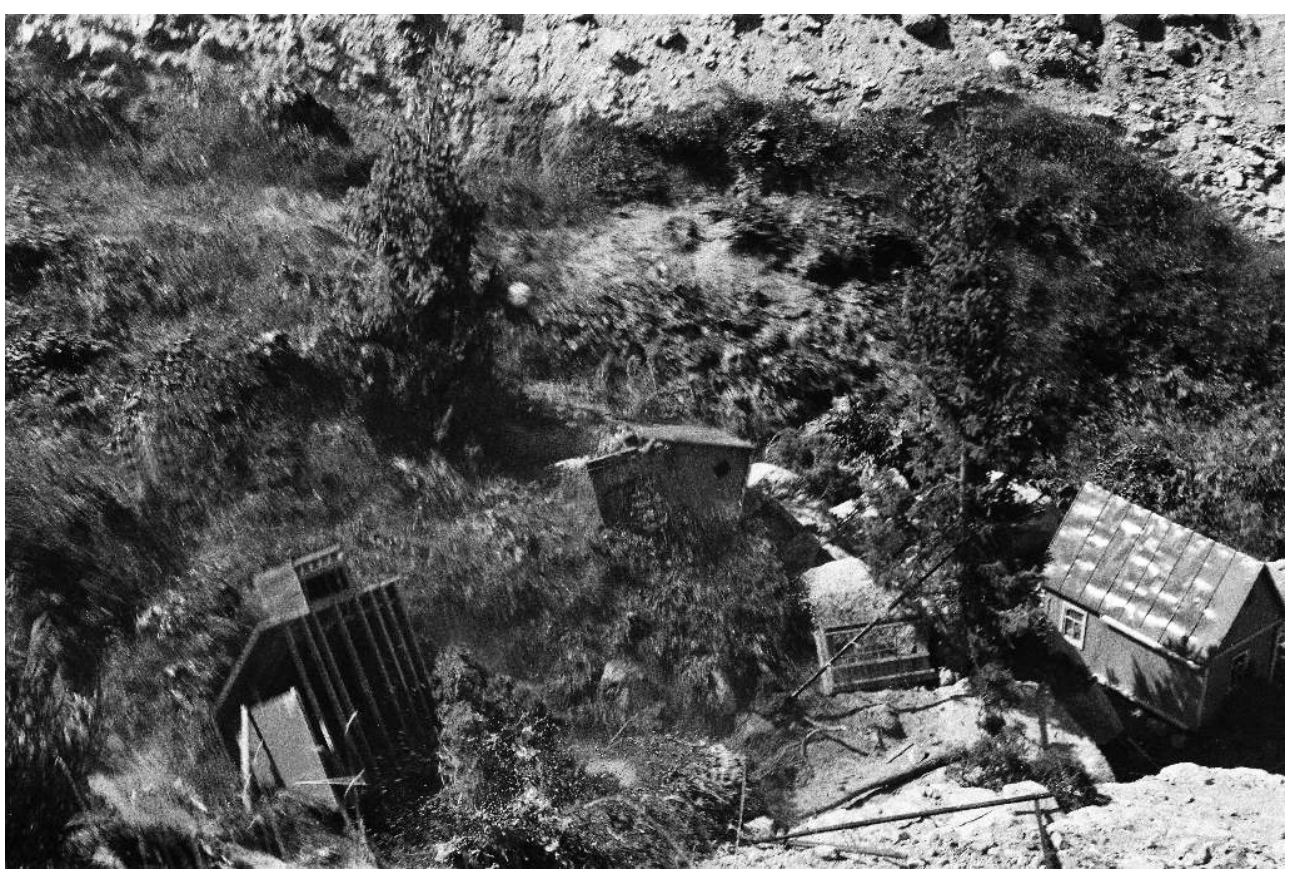

Les maquettes sont emportées par la coulée.

Don d'Alim Hajdarov, archives personnelles, 1978.

36 Nugmanov intégra les images tournées l'année précédente à Čemolgan à son documentaire. Contrairement au film de Kulakov, l'image et le bruit de la coulée étaient fidèlement reproduits : Nugmanov avait utilisé le son enregistré par les hydrologues. 
La qualité de ces images compense quelque peu la faible qualification technique de la voix hors-champ, Nugmanov n'ayant pas fait appel à un conseiller. Pourtant, le réalisateur ne se contenta pas d'inclure telles que ces images et le terrifiant grondement. Il réalisa un montage très simple pour dramatiser l'enregistrement scientifique. D'une durée d'une minute trente secondes, la séquence entrecoupe des plans créés par Nugmanov et les prises de vue de la coulée dévalant les pentes, filmée par les scientifiques. La séquence s'ouvre sur un projecteur déchirant l'obscurité par un signal de détresse. Le désagréable clignotement agresse l'œil du spectateur. Puis on voit en gros plan une montre-bracelet au verre brisé dont les aiguilles arrêtées marquent 18 heures 12 minutes, heure de l'arrivée de la coulée dans le réservoir. On pense à cette horloge arrêtée dans le séisme, à la fois indicatrice de l'heure fatidique et signe de la fin des temps. Un travelling optique sur la montre accentue l'oppression [Ill. 3]. Enfin, la caméra tournoie en contre-plongée sur des épicéas. Obscurité, succession et répétition accélérées d'images, agressivité du projecteur devaient créer une atmosphère oppressante puis vertigineuse, qui cassait la majestueuse progression de la coulée.

En un sens, le montage plongeait le spectateur dans une atmosphère qui n'était pas celle du 15 juillet 1973 : la coulée avait eu lieu en plein jour et aucun signal de détresse n'avait été donné, car le système d'alerte n'avait pas fonctionné ${ }^{52}$. Mais c'est l'effet d'angoisse que visait Nugmanov. La musique inquiète et le discours alarmiste du narrateur soulignaient danger et destruction : " malheur à ce qui se trouve sur son passage... L'eau furieuse engloutit rochers et boue et arrache les arbres, abat les montagnes ». Les effets spéciaux et l'enchaînement avec les travellings sur la station d'alpinisme détruite faisaient apparaître la coulée comme un phénomène extrêmement dangereux, amenant le malheur, et non pas comme un surprenant phénomène naturel digne d'observation et d'admiration, comme l'avaient interprété les hydrologues.

Cependant, tous les films du présent corpus suivent la même structure narrative : d'abord est présentée la capitale kazakhstanaise en harmonieuse interaction avec son écrin alpin. La coulée intervient pour rompre cette alliance : des forces alpestres excessives et incontrôlées apportent la désolation à la civilisation. L'union des avancées technoscientifiques et de la volonté politique rétablit l'harmonie en posant de fermes et claires limites aux puissances naturelles, sous la forme d'un barrage protecteur. Ainsi, le documentaire Medeo. Jours et nuits de courage et la fiction Un bouclier pour la ville suivent le même déroulé.

\section{Le salut dans la catastrophe : À la rescousse du « film de production»"}

L'expérimentation des hydrologues intéressait aussi les réalisateurs de fiction. Quand Leonid Agranovič (1915-2011) tourna Un bouclier pour la ville, en 1978, il mit en scène un ingénieur cherchant contre vents et marées à convaincre habitants, collègues, collaborateurs et responsables de la nécessité de construire un barrage pour protéger la ville d'Alma-Ata contre les coulées de boue ${ }^{53}$. Par un pusillanime renversement du poids politique réel des opposants et avocats au barrage géant à Medeo, le film présente les seconds comme un groupe marginal, réduit à l'ingénieur Kasym Batyrov (Anuarbek Moldabekov) et un adjuvant tardivement gagné à la cause. Les premiers, les opposants au barrage, sont collectivement figurés dans le personnage imposant de l'académicien Aleksandr Aleksandrovič Bortko. De plus, les grincheux ont avec eux la lenteur 
bureaucratique, les conflits d'intérêts entre ministères et l'insouciance des habitants : tous conjurent contre le succès du barrage proposé par Batyrov. Surtout, on prête aux sceptiques un entregent prodigieux dans les milieux gouvernementaux : Bortko est réputé agir dans les coulisses pour casser le projet de barrage, alors que Batyrov et son compère n'ont à leur disposition que les armes honnêtes de la discussion et de la conviction. Ils sont bien seuls, même leur hiérarchie semble les lâcher ; jusqu'à l'apparition tardive d'un conciliateur qui tranche entre Batyrov et Bortko au profit du premier : le "président de la commission gouvernementale». Ce mystérieux personnage n'est autre que Dinmuhamed Kunaev, le premier secrétaire de la république kazakhstanaise. Cette référence est transparente pour le public de l'époque. De même, l'académicien Bortko, chef de file des opposants, est en fait l'académicien V.P. Zaharov. Enfin, Batyrov est Aleksej Hegaj, ingénieur en chef du barrage puis directeur adjoint de Kazglavselezaščita ${ }^{54}$.

Un bouclier pour la ville est visiblement une commande politique. Les studios Kazahfil'm appelèrent Agranovič de Moscou pour écrire un scénario et réaliser un film sur le barrage dans les cadres narratifs du « film à thématique productive » (proizvodstvennyi $\left.f_{i} l^{\prime} m\right)^{55}$. Deux ingénieurs haut placés dans la hiérarchie républicaine furent affectés au tournage en qualité de consultants : R.S. Simonian, directeur de Kazvzryvprom, qui avait conseillé Kulakov pour L'explosion créatrice et Aleksej Hegaj lui-même ${ }^{56}$. Agranovič avait déjà une solide carrière de scénariste et s'était plus récemment lancé comme réalisateur, avec une prédilection pour les drames policiers et les films de production ${ }^{57}$. L'une des conventions de ce dernier genre est de présenter un responsable de niveau moyen (directeur de kolkhoze, ingénieur, responsable d'une cellule du parti) dans sa lutte pour imposer une innovation à une base démotivée, à une hiérarchie lointaine et méfiante, et à des bureaucrates timorés qui freinent des quatre fers. Ici, l'ingénieur Batyrov. En fait, Kunaev lui-même, que le film décrit faussement comme un arbitre neutre intervenant sur le tard, avait tenté d'imposer le barrage dès la fin des années 1950. Surtout, les opposants au barrage ont été une minorité d'abord réduite aux armes de la pétition et de l'indignation, puis muselée par le pouvoir ${ }^{58}$. Quant à Batyrov, il a bien des points communs avec Hegaj : tous deux ont été très marqués par leur expérience d'une coulée de boue réelle ${ }^{59}$. Mais Hegaj est de la minorité coréenne au Kazakhstan. Dans le cadre de la " politique nationale » dans les années 1970, le héros du film ne peut être que Kazakh, représentant de la nationalité titulaire ${ }^{60}$. Pour équilibrer le tableau, Agranovič lui a associé un adjuvant, slave bien sûr.

41 La réception du film fut mitigée. Il faut dire que Batyrov faisait un drôle de héros de la production socialiste : jeté dans des affres terribles, poursuivi par la mort de sa fille, incapable de s'exprimer en public, lâché par ses ouvriers, humilié par les journalistes, il n'exista que grâce à l'intervention finale du bienfaiteur, le "président de la Commission gouvernementale ». Celui-ci fit naître Batyrov à la parole publique, au cours de l'une de ces fameuses réunions de service qui sont au cœur des « films à thématique productive». Certes, Un barrage pour la ville reçut un accueil assez chaleureux dans la république kazakhstanaise, mais il pouvait difficilement en être autrement ${ }^{61}$. Cependant le critique moscovite Armen Medvedev le massacra dans les pages de la Pravda : " On ne trouve pas de héros dans ce film, de personnalité apte à exprimer l'ampleur et la perspective du drame $»^{62}$. Il jugeait le film d'Agranovič bien inférieur aux documentaires sur la construction du barrage ci-dessus évoqués : L'explosion créatrice, Medeo. Jours et nuits de courage, et Le domptage du dragon noir. L'échec 
de la fiction d'Agranovič symbolisait la décadence du " film à thématique productive " pour Medvedev. Le genre avait atteint son apogée au milieu des années 1970, avec le très remarqué La Prime (Sergej Mikaèljan, 1974). Depuis, la qualité des films diminuait, diagnostiquait Medvedev. À moins que la "thématique productive», qui fournissait des scénarios de plus en plus attendus et évoquait l'ennui des réunions de service, n'ait tout simplement fini par lasser le public - hypothèse que n'osait formuler Medvedev.

Toutefois, Agranovič n'avait pas voulu réaliser un pur film à thématique productive. Un barrage pour la ville est un film catastrophe qui s'ouvre sur une coulée de boue qui bouleverse la vie des Batyrov. Kasym, sa femme et leurs deux enfants passent un jour d'été lumineux au bord d'un lac de montagne. Baignade, pique-nique, jeux, rires. Mais dans les hauteurs, une moraine craque bientôt sous le poids de l'eau fondue du glacier : c'est le départ d'une coulée qui anéantit le lac où batifolent les Batyrov. Kasym assiste impuissant à la mort de sa fille. Son fils, grièvement blessé, en réchappe de justesse. Sa femme est inconsolable. Lui-même restera accablé jusqu'à la fin du film, poursuivi dans ses cauchemars par les scènes du désastre et anxieux pour la santé de son fils convalescent.

Le spectateur kazakhstanais reconnaît dans cette scène d'ouverture un épisode historique particulièrement saisissant : la disparition du lac d'Issyk, emporté par une coulée un week-end de l'été 1963. Des dizaines de vacanciers, bronzant sur les berges ou ramant sur le lac, avaient disparu dans le cataclysme : la coulée siphonna le lac dans la rivière d'aval. L'inondation avait sévèrement endommagé la bourgade d'Issyk, située à une cinquantaine de kilomètres d'Alma-Ata ${ }^{63}$. Les médias évoquaient régulièrement la catastrophe. Cependant, il ne s'agissait jamais de la catastrophe humaine, des touristes engloutis dans la boue ou des riverains noyés dans l'eau du lac. L'attention était portée sur la disparition du lac et sur les moyens techniques pour le restaurer : une gigantesque explosion pourrait permettre de le reformer, assuraient les artificiers du barrage de $\mathrm{Medeo}^{64}$.

Un bouclier pour la ville présente Issyk comme un cataclysme pour l'individu et la famille, et non pas comme un défi pour les ingénieurs. Certes, Agranovič n'a pas cherché à reconstituer fidèlement la catastrophe : Il ne fait que l'évoquer via un destin, celui des Batyrov. Ils sont seuls à s'ébaudir au bord du lac, alors qu'à Issyk, des foules de plaisanciers étaient agglutinées autour des eaux turquoise. Contrairement au topo du film catastrophe, le spectateur kazakhstanais ne voit pas dans Un bouclier pour la ville de scène de panique collective, où dans l'affrontement sont révélés les sentiments et les caractères les plus abjects et les plus nobles. Il reste dans l'intimité d'un drame familial, celui des seuls Batyrov. Les interminables discussions dans les cabinets ministériels pâlissent en comparaison du choc initial de la coulée. Le public kazakhstanais a trouvé dans Un bouclier pour la ville la première évocation des malheurs d'Issyk.

Comme les documentaristes avant lui, Agranovič mit à profit les expérimentations scientifiques de Vinogradov pour obtenir les impressionnantes images qui font l'intérêt d'Un bouclier pour la ville. Agranovič fit mieux que Nugmanov, cependant : il négocia avec les hydrologues en amont pour tourner lui-même la scène qui l'intéressait dans l'expérience des scientifiques ${ }^{65}$. Il fit monter des maisonnettes dans la vallée de Čemolgan. Dix caméras tournaient, dont une depuis un hélicoptère et une autre au niveau du lit du torrent filmait de face la coulée anéantissant les maisonnettes ${ }^{66}$. Les images obtenues devinrent le principal matériau utilisé par Agranovič pour évoquer la catastrophe qui frappa les Batyrov [Ill. 6 et 7]. 
Au moment où Agranovič tourne Un bouclier pour la ville, le film catastrophe connaît ses premiers succès en URSS. Traditionnellement, le cinéma soviétique montre rarement des scènes de catastrophes naturelles ou technologiques se déroulant sur le territoire soviétique. On redoute de démoraliser. La nature est présentée comme maîtrisée, sauf à mettre en scène des pays étrangers ou des époques révolues. Ainsi, jusqu'à la fin des années 1970, les films catastrophes soviétiques ne se déroulent pas en URSS. Le fameux 713-j prosit posadku [Le vol 713 demande l'autorisation d'atterrir] (Grigorij Nikulin, Lenifil'm, 1962), l'un des premiers rôles de Vladimir Vysockij, se déroule à bord d'un avion de ligne étranger. Sorti en pleine " crise des missiles ", il met en scène des passagers dont pas un seul n'est soviétique. Krasnaja palatka [La tente rouge], une coproduction italo-russe réalisée par Mihail Kalatozov en 1969, narre le sauvetage d'un dirigeable italien lors d'une expédition polaire de l'aviateur Umberto Nobile. La catastrophe ne se déroule pas en Union soviétique et les acteurs de cette catastrophe ne sont pas citoyens soviétiques, à l'exception de l'infirmière Valeria incarnée par Claudia Cardinale.

La fin des années 1970 voit un intérêt croissant pour les films catastrophes. Commission d'enquête (Komissija po rassledovaniju, Vladimir Bortko, Lenfil'm, 1978) relate les luttes d'intérêt entre les représentants des institutions chargées d'investiguer une panne dans un réacteur nucléaire de type RBMK (le même que les réacteurs de Černobyl). La catastrophe est évitée dès le début du film, qui n'a pas le danger et l'urgence pour sujets. Les cadres $\mathrm{du}$ " film de production" ne sont pas dépassés. Le changement intervient avec Équipage (Aleksandr Mitta, Mosfil'm, avec Georgij Žženov, musique d'Al 'fred Šnittke, 1979). Grand succès auprès du public, Équipage met en scène dans une première partie les péripéties de carrière et de la vie privée des pilotes (divorces et mutations). Dans une seconde partie, l'équipage enfin constitué prend part à une mission de sauvetage dans un pays du tiers-monde : une cité pétrolière est frappée par un tremblement de terre et une gigantesque coulée de boue. L'avion parvient à évacuer les coopérants soviétiques, mais il est tellement endommagé que le voyage du retour manque de se terminer en désastre. Face au triomphe hollywoodien d'Airport (George Seaton, 1970), de La Tour infernale (The Towering Inferno, John Guillermin et Irwin Allen, 1974), de L'aventure du Poséidon (The Poseidon Adventure, Ronald Neame, 1972) et de Tremblement de terre (Earthquake, Mark Robson, 1974), qui ne sont pas diffusés en URSS, le cinéma soviétique cherche le succès commercial dans les films à suspense mettant en scène des gens simples placés dans des situations extrêmes.

Un bouclier pour la ville s'inscrit dans le glissement vers des films spectaculaires à gros budget où la contemplation cathartique du désastre (train en flammes, ouragans) ${ }^{67}$ le dispute à la thématique traditionnelle de l'héroïsme collectif. L'action est prise en sandwich entre la scène de la catastrophe lacustre chez les Batyrov et l'inquiétude de la voir élargie à toute la ville, créant un sentiment d'urgence. Un bouclier pour la ville introduit une tension provenant d'une menace extérieure. La thématique catastrophique était perçue comme apte à renouveler l'intérêt du public pour des films sur les realia soviétiques hors mélodrame et comédie, dans le genre " sérieux " des "films à thématique productive " mettant en scène des gens au travail (par exemple des pilotes dans un avion en perdition), et même plus largement portant sur la " réalité soviétique» (sovremennaja žizn', sovetskaja sovremennost'). Ce renouvellement avait une conséquence importante : les cinéastes pouvaient désormais évoquer des catastrophes technologiques et naturelles en URSS, et ils ne s'en privèrent pas ${ }^{68}$. 
La fiction d'Agranovič devait donner une image lisse de l'histoire de la construction du barrage. Le film sédimentait les chaînes causales en construisant un modèle action réaction classique : la catastrophe est première, les hommes doivent y répondre par le progrès technique sous la tutelle d'une bureaucratie technoscientifique supervisée par un leader politique. Les interrogations écologiques et sociales, telles qu'on peut les entendre dans Les Adieux à Matiora d'Elem Klimov (1981), et dans le documentaire Le journal de Naryn d'Al'gimantas Vidugiris ${ }^{69}$ (1971), sur les conséquences des grands travaux d'aménagement étaient évacuées. Le contraste est intéressant avec le film catastrophe hollywoodien qui cherchait au contraire à attribuer les responsabilités humaines, de la négligence criminelle à l'acte maléfique ${ }^{70}$. Le film catastrophe soviétique paraît par contraste idéologiquement apaisé, désidéologisé même, une caractéristique déjà soulignée de la production culturelle sous Brežnev ${ }^{71}$.

50 Toutefois, les films soviétiques qui thématisent la catastrophe restèrent rares. Ils ne s'émancipaient pas complètement des conventions narratives du drame et du film de production, même si le suspense jouait un rôle central. Alors que les studios états-uniens et japonais produisaient des centaines de films catastrophes dans les années de guerre froide, on en compte une dizaine en URSS ${ }^{72}$. Surtout, la catastrophe nucléaire était encore plus rarement thématisée, là aussi une différence de taille avec Hollywood et les studios japonais. Les plus célèbres romans de science-fiction mobilisant l'explosion nucléaire, La Nébuleuse d'Andromède d'Ivan Efremov (1957) et L'Île habitée des frères Strugackij $(1969)^{73}$, ne connurent pas d'incarnation complète au cinéma : dans l'adaptation du premier, les scénaristes évacuèrent le suicide nucléaire de la civilisation visitée par les humains, thème central du romancier. L'Île habitée ne fut pas adaptée à l'écran à la période soviétique ${ }^{74}$. Dans les catastrophes cinématographiques soviétiques, leurs effets sur la société et sur les corps sont limités, d'où l'exclusion des armes de destruction massive. Quand ce sont les forces naturelles qui menacent et détruisent, elles n'apparaissent jamais comme entièrement incontrôlables ; elles ne menacent jamais la société, la civilisation, le genre humain ou la planète. L'utopie communiste dans sa version doctrinaire étouffait le mouvement dystopique. À un journaliste qui lui demandait en quoi Un bouclier pour la ville se distinguait des films catastrophes étrangers, Agranovič répondit : "Notre film n'effraie pas en montrant la situation, mais inspire la foi dans la raison humaine, dans sa capacité à tenir en échec les forces destructrices de la nature $»^{75}$. L'effroi était rapidement subjugué par la foi dans le contrôle.

\section{Conclusion}

$51 \mathrm{Au}$ moment où les travaux de Vinogradov sur les laves torrentielles accédaient à la reconnaissance, la situation institutionnelle au Kazakhstan évoluait au désavantage des hydrologues de KazNIGMI. Le dispositif expérimental de Čemolgan était prestigieux, unique en URSS et dans le monde ${ }^{76}$. Une agence gouvernementale kazakhstanaise de lutte contre les coulées de boue, Kazglavselezaščita convoitait le "polygone expérimental » de Čemolgan. Fondée en 1973 pour reconstruire le barrage endommagé par la coulée, cette agence de travaux publics était entièrement dans les mains du premier secrétaire Kunaev qui investit financièrement, humainement et logistiquement pour la doter des moyens et des ressources dernier cri qui la mettraient à l'avant-garde de l'innovation en ingénierie d'ouvrages d'art, de systèmes de prévision 
et prévention des risques de montagne et de réseaux d'alarme. Si, sur le papier, la division des tâches entre ingénieurs de Kazglavselezaščita et chercheurs hydrologues du KazNIGMII était claire, l'expérimentation de Vinogradov intéressait les premiers au plus haut point : ils espéraient pouvoir y tester des ouvrages d'art destinés à la prévention des coulées ${ }^{77}$. Par ailleurs, les hydrologues critiquaient dans les périodiques spécialisés les méthodes explosives des artificiers de Kazglavselezaščita dans la vidange préventive des lacs glaciaires, n'hésitant pas à les accuser de déclencher accidentellement des coulées ${ }^{78}$. Vinogradov dut quitter son poste et le Kazakhstan en 1978 pour une chaire d'hydrologie à Leningrad. Kazglavselezaščita accapara le dispositif expérimental de Čemolgan. Le laboratoire de Vinogradov fut vidé de ses forces, happées par la nouvelle agence ${ }^{79}$. Pourtant, celle-ci ne parvint pas à reprendre à un niveau sérieux les expérimentations ${ }^{80}$. La forteresse expérimentale de Vinogradov tomba sous les coups du pouvoir politique. Ce fut la fin brutale des recherches sur les coulées au Kazakhstan soviétique.

Restent les images tournées par les hydrologues au Kazakhstan. Au-delà de la justification du barrage, les réalisateurs ont donné leur interprétation du phénomène des coulées. Du film factuel, les images sont passées au documentaire, puis à la fiction dans l'un des premiers films catastrophes soviétique. À chaque passage, les réalisateurs ont réinterprété au montage le matériau de l'expérimentation : en plongeant le spectateur dans des univers de perception, ils ont fait de la coulée tantôt un phénomène élégant, sublime et enthousiasmant, tantôt une calamité rappelant les dévastations de la guerre, tantôt un drame pour l'individu. Le son et l'image de l'expérimentation de Čemolgan ont fait le tour des médias filmés au Kazakhstan. Tous les participants y voyaient un matériau susceptible de devenir au montage un vecteur pour frapper émotionnellement le public. La recherche de moyens et de modèles cinématographiques nouveaux poussa les cinéastes à explorer la thématique catastrophiste et ses potentialités visuelles, suscitant une rupture dans les cadres d'expression et de réception, puisqu'on pouvait désormais filmer à grand renfort d'effets spéciaux et de décorations spectaculaires des situations de crise dans l'URSS en temps de paix.

53 L'introduction des rushes tournés par les hydrologues a contribué à pluraliser les visions des phénomènes naturels dangereux. Les images des coulées artificielles utilisées dans les documentaires et la fiction donnaient à voir un phénomène unique et grandiose, digne de craintive admiration. Ce changement conduisit les réalisateurs à développer l'esthétique du sublime devant les montagnes sauvages au lieu de recourir à la rhétorique martiale de la lutte contre les éléments encore centrale chez Kulakov. Même si ces films étaient des projets de la direction kazakhstanaise et servaient un scénario optimiste simpliste, la pluralité des interprétations ouvertes par les mises en scène catastrophiques nuançait la rhétorique sur la « domination de la nature » que le barrage géant de Medeo avait contribué à imposer dans les discours officiels ${ }^{81}$. C'est une nature alpestre dangereuse mais fascinante, irréductible aux savoirs scientifiques et échappant aux manipulations techniques qui est mise en avant. Les matériaux cinématographiques produits par les hydrologues ont permis aux réalisateurs de renouveler l'esthétique de la nature dans ses manifestations catastrophiques. 


\section{NOTES}

1. V.D. Gorodeckij, Pričiny Alma-Atinskoj katastrofy 8 ijulija 1921 g. [Les causes de la catastrophe d'Alma-Ata du 8 juillet 1921], $3^{\mathrm{e}}$ édition (Alma-Ata : Obščestvo izučenija Kazahstana, 1936).

2. Cet article utilise " coulée de boue " comme synonyme vulgaire du terme technique "lave torrentielle». En russe, une distinction proche existe entre le terme courant " sel'» et l'expression scientifique « grjazekamennyj potok».

3. Marc Elie, "Coping with the "Black Dragon". Mudflow Hazard and the Controversy over the Medeo Dam in Kazakhstan, 1958-1966», Kritika : Explorations in Russian and Eurasian History, 14 (2), 2013, p. 313-342.

4. Marc Elie, "Gouverner par les aléas : maîtrise des coulées de boue et mise en valeur touristique des montagnes à Alma-Ata (Kazakhstan), 1966-1977 ", in Julien Langumier, Sandrine Revet, éds., Le Gouvernement des catastrophes, P. : Karthala, 2013, p. 33-72.

5. L'auteur n'a pas eu accès aux archives du studio Kazahfil'm. Les trois documentaires sont conservés aux Archives publiques centrales des documents cinéma, photo et audio de la république du Kazakhstan (CGA Kinofotodokumentov i zvukozapisi RK) : Asylbek Kulakov, Vzryv sozidatel' [L'énergie créatrice], 50 min. (Studio Kazahfil'm, 1970), sous le n 3275 ; A. Nugmanov, Medeo. Dni i noči mužestva [Medeo. Jours et nuits de courage], 20 min. (Studio Kazahfil'm, 1973), sous le $n^{\circ} 3202$; et A. Nugmanov, Ukroščenie Černogo drakona [Le domptage du dragon noir], 20 min. (Studio Kazahfil'm, 1976), sous le $n^{\circ}$ 3635. Le film de Leonid Agranovič, Ščit goroda [Le bouclier de la ville], 1979, également produit par le studio Kazahfil'm, a pu être visionné aux Archives de films de la Fédération de Russie (Gosfil' mofond). Le dossier papier du film, contenant entre autres le relevé du commentaire (montažnaja zapis') et le premier scénario écrit par Agronovič, y est conservé au fond 3, opis' 1, delo 3909 (noté dorénavant f. 3, op. 1, d. 3909).

6. Le professeur Boris Stepanov de l'Institut d'hydrométéorologie du Kazakhstan (KazNIGMI), vétéran des expérimentations à Čemolgan, que je remercie ici, m'a remis une copie du film scientifique produit par les hydrologues : V. Serbo, Slovo o selevom potoke [Le Dit de la coulée de boue], 19 mn., produit par KazNIGMI, Alma-Ata, 1979.

7. Bruno Latour, La science en action : introduction à la sociologie des sciences, P. : La Découverte/Poche, 2005, p. 249.

8. Irina Tcherneva, «Imiter le marché, une recette pour le cinéma soviétique ? L'histoire du Studio artistique expérimental (1965-1976) », Cahiers du Monde russe, 54 (3-4), 2013, p. 589-621, ici p. 590.

9. GARF (Gosudarstvennyj arhiv Rossijskoj Federacii - Archives d'état de la Fédération de Russie), f. R-5446, op. 25, d. 3801, 1. 57.

10. Minutes de la réunion pour la coordination des recherches sur les coulées de boue, 23-24 février 1949, ARAN (Arhiv Rossiiskoj Akademii Nauk - Archives de l'Académie des Sciences de Russie), f. 535, op. 1, d. 119, 1. 1.

11. Ibid., 1. 29-30.

12. «... [L]es coulées sont essentiellement des flux d'eau... Pour cette raison, c'est précisément l'hydrologie qui doit étudier [leur] occurrence, leur mécanique et ... les conditions de leur formation...». Irina Bogoljubova, Selevye potoki i ih rasprostranenie na territorii SSSR [Les coulées de boue et leur répartition sur le territoire de l'URSS] (L. : Gidrometeorologičeskoe izdatel'stvo, 1957), p. 32.

13. Esen Dujsenov, « Selezaščitnye meroprijatija v Zailijskom Alatau (Respublikanskaja komissija po bor'be s seljami, Alma-Ata) [Les mesures anti-coulée dans l'Alatau Trans-Ili

(La commission républicaine de lutte contre les coulées à Alma-Ata)]», in H.A. Ahmedžanov, éd., 
Voprosy izučenija selej [Problèmes de l'étude des coulées], M.: Moskovskoe otdelenie gidrometeoizdata, 1969, p. 73-82, ici p. 74.

14. Ou bien un piano à queue par une coulée sur trois kilomètres sur la rivière Durudži (Géorgie) en 1949. Materialy IV vsesojuznoj konferencii po selevym potokam, Alma-Ata 19-23 nojabrja 1956 [Actes du $4^{\mathrm{e}}$ congrès pan-soviétique sur les coulées, Alma-Ata 19-23 novembre 1956], Alma-Ata : Izdatel'stvo Akademii nauk Kazahskoj SSR, 1959, p. 210.

15. Minutes de la réunion pour la coordination des recherches sur les coulées de boue, 1. 29-30.

16. Materialy IV vsesojuznoj konferencii, p. 209.

17. T. Bajmoldaev, V. Vinohodov, Kazselezaščita - Operativnye mery do i posle stihii [L'agence Kazselezaščita : action avant et après le cataclysme], Almaty : Bastau, 2007, p. 39.

18. S.P. Kaveckij, "Seli v Zailijskom Ala-Tau [Les coulées dans l'Alatau Trans-Ili]», Trudy KazNIGMI [Travaux de KazNIGMI], 1953, 7-38.

19. Semen Moiseevič Flejšman, Seli [Les coulées de boue], $2^{\mathrm{e}}$ édition, L. : Gidrometizdat, 1978, p. 6-7.

20. La difficulté de l'observation est commune aux phénomènes rares et sporadiques, comme les météores. Fabien Locher, " Le Nombre et Le Temps. La Météorologie En France (1830-1880) », thèse de doctorat, EHESS, 2004.

21. Kulakov, Vzryv sozidatel'.

22. Elie, « Gouverner par les aléas », p. 39.

23. From the KTLA Archive : Baldwin Hills Dam Collapse Disaster <http://ktla.com/2013/12/12/ from-the-ktla-archive-baldwin-hills-dam-collapse-disaster/> [consulté le 2 avril 2014].

24. Elie, " Coping with the "Black Dragon" ", p. 334.

25. Semen Flejšman, Seli, p. 177 précise que la densité moyenne de la coulée est le paramètre central pour en déduire les caractéristiques physiques.

26. B.S. Stepanov, « Ključevaja rol' teorii suščestvovanija selevoj massy pri rasčete harakteristik selja [Le rôle clef de la théorie de l'existence d'un mélange pour le calcul des caractéristiques des coulées] », in Trudy vtoroj konferencii posvjaščennoj 100-letiju so dnja roždenija S.M. Flejšmana. 17-19 oktjabrja 2012 [Travaux du second congrès pour le $100^{\mathrm{e}}$ anniversaire de S.M. Flejšman, 17-19 octobre 2012], M. : Geografičeskij fakul'tet MGU, 2012, pp. 84-85.

27. L.P. Smirnov, « Opyt izučenija selej putem iskusstvennogo ih vosproizvedenija [Pratique de l'étude des coulées via leur production artificielle]», Trudy KazNIGMI, 1953, pp. 39-53, ici p. 46.

28. S.P. Kaveckij, Grjazekamennyj potok [Les laves torrentielles], 17 min., produit par le Cabinet cinématographique de l'Académie des sciences du Kazakhstan, Alma-Ata, 1952. Alim Hakimovič Hajdarov, ancien technicien au laboratoire des coulées de KazNIGMI, vétéran de l'expérimentation de Čemolgan, photographe et archiviste, m'a remis une copie de ce film. Je le remercie ici.

29. T.S. Stepanova, «U istokov selevoj nauki v Kazahstane. S.P. Kaveckij, I.P.Smirnov. K 100-letiju so dnja roždenija S.P. Kaveckogo [À l'origine de la science des coulées au Kazakhstan. S.P. Kaveckij, I.P.Smirnov. Pour le $100^{\mathrm{e}}$ anniversaire de S.P. Kaveckij]», Gidrometeorologija i èkologija, 2012, p.179-193, ici p.184. Pour une raison inconnue, ces expériences furent interrompues dès 1953.

30. R.V. Honin, « Issledovatel' selevyh potokov Ju.B. Vinogradov [Ju.B. Vinogradov, chercheur en coulées de boue] ", Mednyj vsadnik. Kazahstan, 2004, p. 31-39, ici p. 32 ; Ju. B. Vinogradov, Ètjudy o selevyh potokah [Études sur les coulées de boue], L. : Gidrometizdat, 1980, p. 70-71.

31. Ju.B. Vinogradov, « Iskusstvennoe vosproizvedenie selevyh potokov na èksperimental'nom poligone $\mathrm{v}$ bassejne $\mathrm{r}$. Čemolgan [La production artificielle de coulées sur le site expérimental du torrent Čemolgan] », Selevye potoki, 1, 1976, p. 3-7.

32. Telle que la formulait Simen Moiseevič Flejšman (1912-1984), président de la Commission des coulées dans les années 1970.

33. Vinogradov, « Iskusstvennoe vosproizvedenie », p. 4. 
34. R.V.Honin, V.P. Močalov, A.È. Zems, « ̇̀skperimental'nyj poligon v bassejne r. Čemolgan i istorija ego sozdanija [Le site expérimental du torrent Čemolgan et l'histoire de sa création] ", Selevye potoki, 1, 1976, p. 7-25, ici p. 9.

35. Honin, «Issledovatel' selevyh potokov Ju.B. Vinogradov », p. 31 et 39.

36. Ju.B. Vinogradov, directeur du secteur des coulées de boue [de KazNIGMI], « Programme de l'expédition pour la réalisation de coulées de boue artificielles sur la rivière Čemolgan". 1.02.1972. CGANTD (Central'nyj gosudarstvennyj arhiv naučno-tehničeskoj dokumentacii Kazahstana - Archives publiques centrales de la documentation scientifique et technique du Kazakhstan), f. 121m, op. 1-6, d. 447, 1. 16-20.

37. Jurij Vinogradov, commentant la coulée en voix hors-champ, dans le film Slovo o selevom potoke.

38. Latour, La science en action.

39. Honin, Močalov, Zems, « Èskperimental'nyj poligon»; Bruno Latour, "Les "vues" de l'esprit », Réseaux, 5, p. 79-96.

40. Jurij Vinogradov, Gljacial'nye proryvnye pavodki i selevye potoki [Crues glaciaires irruptives et coulées de boue], M. : Gidrometizdat, 1977, p. 4-5.

41. A. Degovec, ingénieur hydrologue à Kazgidroproekt, "Rapport d'étude sur la coulée expérimentale provoquée par KazNIGMI dans les conditions naturelles du bassin du torrent Čemolgan le 27 août 1972 ", non daté [1972], CGAgA (Central'nyj gosudarstvennyj arhiv goroda Almaty - Archives centrales de la ville d'Almaty), f. 174, op. 29, d. 1206, 1. 180-189, ici 185.

42. A.È. Zems, R.V. Honin et V.I. Laptev, « Balans tverdogo i židkogo veščestva v selevyh potokah 1972-1973 gg. v bassejne r. Čemolgan [Bilan de la matière solide et liquide dans les coulées de 1972-1973 dans le torrent Čemolgan]», Selevye potoki, 1, 1976, p. 35-43.

43. Degovec, « Rapport d'étude».

44. Elie, « Gouverner par les aléas », p. 44 et 53.

45. Honin, «Issledovatel' selevyh potokov Ju.B. Vinogradov », p. 37 ; Ju.B. Vinogradov, A.È. Zems, R.V. Honin, « Selevoj potok 15 julija 1973 g. na Maloj Almatinke » [La coulée du 15 juillet 1973 sur la Petite Almatinka], in Selevye potoki, L. : Gidrometizdat, 1976, p. 60-73.

46. Outre la densité, calculer le débit de la coulée à partir des traces de la coulée était un exercice périlleux : Smirnov le surestima en partant de la surface de la section transversale de la coulée dans le lit du torrent. Contrairement à l'intuition, plus cette surface est grande, moins la vitesse est rapide et le débit fort, car la coulée s'étale en s'arrêtant. Vinogradov, Gljacial'nye proryvnye pavodki i selevye potoki, p. 57.

47. Roza Jafjazova, Priroda selej Zailijskogo Alatau : Problemy adaptacii [La nature des coulées dans l'Alatau Trans-Ili : les défis de l'adaptation], Almaty, 2007, p. 107.

48. Ce n'est que lors de la seconde expérience l'année suivante que Stepanov put éprouver son débitmètre spécialement conçu pour les coulées.

49. Entretien avec Boris Sergeevič Stepanov, Almaty, 24 juin 2013 [notes manuscrites].

50. Nugmanov, Medeo. Dni i noči mužestva.

51. Gestwa Klaus, Die Stalinschen Großbauten des Kommunismus : Sowjetische Technik- und Umweltgeschichte, 1948 - 1967, München : Oldenbourg, 2010, p. 56-57.

52. Elie, « Gouverner par les aléas », p. 43.

53. Agranovič, Ščit goroda.

54. Entretien avec Aleksej Hegaj, Almaty, 22 mai 2010 [enregistrement numérique].

55. Gosfil'mofond, f. 3, op. 1, d. 3909.

56. « Ščit goroda. Interview avec L. Agranovič », Novyj fil’m, n² 22, 1978, cité dans Aleksej Jur 'evič Hegaj, Vremeni bystraja reka... [Du temps le cours rapide...], Alma-Ata : Kazahstan, 1999, p. 47-48. 
57. «Skončalsja scenarist, režisser i dramaturg Leonid Agranovič »[Décès du scénariste, réalisateur et dramaturge Leonid Agranovič], RIA Novosti, $2011<$ http://ria.ru/culture/ 20110315/354198802.html> [accessed 13 March 2015].

58. Elie, " Coping with the "Black Dragon" ", p. 339.

59. Aleksej Hegaj, Ukroščenie "Černogo drakona », (Alma-Ata : Kazahstan, 1988). Cependant, Aleksej Hegaj n'a joué aucun rôle dans la promotion du barrage lors des débats des années 1960 .

60. Entretien avec Hegaj.

61. N. Tjutjavina, « Devjatyj val stihij [La neuvième vague du cataclysme] », Večernjaja Alma-Ata, 26 décembre 1979, p. 3. Le film fut primé à deux reprises : il reçut un "diplôme d'honneur » pour son « élaboration du thème de la classe ouvrière » à Duchanbe (Tadjikistan) en 1980 lors du festival du film soviétique (VKF). Et plus étonnamment, au festival du film pour enfant et pour la jeunesse de Gijon en Espagne en 1979.

62. Armen Medvedev, « Protokol zasedanija ili istorija haraktera? Zametki o proizvodstvennoj teme na èkrane [Compte-rendu de séance ou histoire d'un caractère ? Notes sur la thématique productive à l'écran] », Pravda, 22 octobre 1980, p. 3.

63. Elie, " Coping with the "Black Dragon" ", p. 329.

64. Elle est mentionnée deux fois dans Kulakov, Vzryv sozydatel'.

65. Les spécialistes se gaussaient de ce décor bien peu réaliste : les maisons, les poteaux électriques et les arbres se dressaient à même le lit du torrent. Entretien avec Stepanov, Amaty, 27 et 28 septembre 2011 [enregistrement numérique] et avec Hajdarov, Almaty, 23 juin 2013 [enregistrement numérique].

66. Selon le récit du chef-opérateur: Galija Šimyrbaeva, "Igor' Vovnjanko: professija «kinošnika» i opasna, i trudna » [Igor' Vovnjanko: cinéaste, une profession dangereuse et pénible], Kazahstanskaja pravda, 7 décembre 2002 <http://kp.kazpravda.kz/print/1002071281> [accessed 13 March 2015].

67. Dans un article célèbre, "Imagination of Disasters", Susan Sontag pose que l'effet cathartique ou empathique des scènes de destruction est typique des films catastrophes. Cité par Susan J. Napier, " Panic Sites: The Japanese Imagination of Disaster from Godzilla to Akira ", Journal of Japanese Studies, 19 (2), 1993, p. 327-351, ici p. 332.

68. Par exemple : 34-j skoryj [L'express 34], Andrei Maljukov, Mosfil'm 1981 ; Štormovoe predupreždenie [Alerte Tempête], Vadim Mihajlov, Lenfil'm, 1981 ; Poezd vne raspisanija [Un train imprévu], Aleksandr Grišin, Odesskaja kinostudija, 1985.

69. Je remercie Moritz Florin d'avoir attiré mon attention sur ce film.

70. Stephen Keane, Disaster Movies: The Cinema of Catastrophe, Columbia University Press, 2001, p. 17.

71. Natalya Chernyshova, "Philistines on the Big Screen : Consumerism in Soviet Cinema of the Brezhnev Era », Studies in Russian and Soviet Cinema, 5 (2), 2011, p. 227-254.

72. Napier, « Panic Sites: The Japanese Imagination of Disaster from Godzilla to Akira » Mick Broderick, "Surviving Armageddon: Beyond the Imagination of Disaster ", Science Fiction Studies, 20 (3), 1993, p. 362-382.

73. Edward Geist, « Two Worlds of Civil Defense : State, Society, and Nuclear Survival in the USA and USSR, 1945-1991 », thèse de doctorat, 2013, p. 196-201. Je le remercie de m'avoir remis une copie du chapitre 2 de sa thèse.

74. Sur la science fiction soviétique de la période poststalinienne voir l'introduction et le numéro dirigé par Istvan Csicsery-Ronay, Jr., " Science Fiction and the Thaw », Science Fiction Studies, 31 (3), 2004, p. 337-344 ; Le processus d'écriture du scénario pour l'adaptation de Pique-nique au bord de la route des Frères Strugackij par Tarkovskij est symptomatique de la difficulté du cinéma soviétique avec la science-fiction. Les Strugackij et Tarkovskij ont mis longtemps à s'entendre sur un texte dans lequel tout élément de science-fiction fut finalement exclu. Stalker est ainsi une "fable spirituelle» (Csicsery-Ronay), mais pas un film de science-fiction. Boris 
Natanovich Strugatsky et Erik Simon, « Working for Tarkovsky », Science Fiction Studies, 31 (3), 2004, p. 418-420.

75. « Ščit goroda. Interview avec L. Agranovič ».

76. En 1977, Vinogradov publia une monographie qui fait le point sur les coulées d'origine glaciaire à forte densité telles qu'il les avait reproduites à Čemolgan. Vinogradov, Gljacial'nye proryvnye pavodki i selevye potoki.

77. Dès 1977, Kazglavselezaščita entendit tester ses barrages à Čemolgan. CGARK (Central'nyj gosudarstvennyj arhiv Respubliki Kazhstana - Archives publiques centrales de la république du Kazakhstan,) f. 1137, op. 27, d. 919, 1. 19-21.

78. Elie, « Gouverner par les aléas », p. 54-57.

79. Honin, «Issledovatel” selevyh potokov Ju.B. Vinogradov », p. 39.

80. L'agence réalisa un lâcher en août 1991 dont les résultats furent décevants. V.P. Močalov, A.K. Kim, A.H.Hajdarov, "Rezul'taty èksperimenta “Čemolgan-91” [Résultats de l'expérimentation « Čemolgan-91»]», Selevye potoki, 12, 1992, p. 101-109.

81. La redécouverte par les cinéastes de la nature comme dépassant les individualités dans les années 1960 est analysée par Marko Dumancic, « De-Stalinizing Soviet Science : Rethinking the Moral Implication of Scientific Progress in Khrushchev-Era Film », Studies in Russian and Soviet Cinema, 6, 2012, p. 75-92.

\section{RÉSUMÉS}

Cet article raconte l'histoire de la mise en image des coulées de boue, phénomènes naturels dangereux et insaisissables, au Kazakhstan soviétique des années 1970-1980. Il croise trois questionnements : 1) comment des hydrologues kazakhstanais cherchèrent à visualiser les coulées pour imposer leur conception de leurs caractéristiques physiques ;2) comment les réalisateurs utilisèrent les rushes de coulées des films scientifiques pour véhiculer un message rassurant à la population sur la capacité des autorités à maîtriser les risques glaciaires ; 3) et comment ces images spectaculaires contribuèrent à la naissance d'un nouveau format narratif au cinéma soviétique, le film catastrophe.

This article tells the story of how mudflows - dangerous and elusive natural phenomena - were filmed in Soviet Kazakhstan in the 1970s-1980s. It asks three questions: 1) How did Kazakh hydrologists succeed in filming mudflows and resolve a scientific controversy on mudflow physics? 2) How did filmmakers use footage created by scientists to propagate a reassuring message on the authorities' ability to control mudflow risks? 3) How did this impressive footage contribute to the birth of a new narrative genre in Soviet cinema, the disaster film?

\section{AUTEUR}

\section{MARC ELIE}

CERCEC EHESS-CNRS, marc.elie@cercec.cnrs.fr 